\title{
Dense trajectories and motion boundary descriptors for action recognition
}

\author{
Heng Wang · Alexander Kläser • Cordelia Schmid • Cheng-Lin Liu
}

Received: date / Accepted: date

\begin{abstract}
This paper introduces a video representation based on dense trajectories and motion boundary descriptors. Trajectories capture the local motion information of the video. A dense representation guarantees a good coverage of foreground motion as well as of the surrounding context. A stateof-the-art optical flow algorithm enables a robust and efficient extraction of dense trajectories.

As descriptors we extract features aligned with the trajectories to characterize shape (point coordinates), appearance (histograms of oriented gradients) and motion (histograms of optical flow). Additionally, we introduce a descriptor based on motion boundary histograms $(\mathrm{MBH})$ which rely on differential optical flow. The MBH descriptor shows to consistently outperform other state-of-the-art descriptors, in particular on real-world videos that contain a significant amount of camera motion.

We evaluate our video representation in the context of action classification on nine datasets, namely KTH, YouTube, Hollywood2, UCF sports, IXMAS, UIUC, Olympic Sports, UCF50 and HMDB51. On all datasets our approach outperforms current state-of-the-art results.
\end{abstract}

Keywords Action recognition - Dense trajectories · Motion boundary histograms

H. Wang · C.-L. Liu

National Laboratory of Pattern Recognition

Institute of Automation, Chinese Academy of Sciences

e-mail: \{hwang,liucl\}@nlpr.ia.ac.cn

A. Kläser · C. Schmid

LEAR team, INRIA Grenoble Rhône-Alpes

655 Avenue de l'Europe 38330 Montbonnot, France

e-mail: \{alexander.klaser, cordelia.schmid\}@inria.fr

\section{Introduction}

Local space-time features are a successful representation for action recognition. Laptev (2005) has introduced space-time interest points by extending the Harris detector to video. Other detection approaches are based on Gabor filters (Bregonzio et al, 2009; Dollár et al, 2005) and on the determinant of the spatio-temporal Hessian matrix (Willems et al, 2008). Feature descriptors range from higher order derivatives (local jets), gradient information, optical flow, and brightness information (Dollár et al, 2005; Laptev et al, 2008; Schüldt et al, 2004) to spatio-temporal extensions of image descriptors, such as 3D-SIFT (Scovanner et al, 2007), HOG3D (Kläser et al, 2008), extended SURF (Willems et al, 2008), and Local Trinary Patterns (Yeffet and Wolf, 2009).

However, the 2D space domain and 1D time domain in videos show different characteristics. It is, therefore, more intuitive to handle them in a different manner than to detect interest points in a joint 3D space. Tracking interest points through video sequences is a straightforward choice. Some recent methods (Matikainen et al, 2009; Messing et al, 2009; Sun et al, 2009, 2010) show good results for action recognition by leveraging the motion information of trajectories. To obtain feature trajectories, either tracking techniques based on the KLT tracker (Lucas and Kanade, 1981) are used (Matikainen et al, 2009; Messing et al, 2009), or SIFT descriptors between consecutive frames are matched (Sun et al, 2009). Recently, Sun et al (2010) combined both approaches and added random trajectories in low density regions of both trackers in order to increase density.

Dense sampling has shown to improve results over sparse interest points for image classification (Fei-Fei and Perona, 2005; Nowak et al, 2006). The same is observed for action recognition in a recent evaluation by Wang et al (2009), where dense sampling at regular positions in space and time outperforms state-of-the-art spatio-temporal interest point de- 

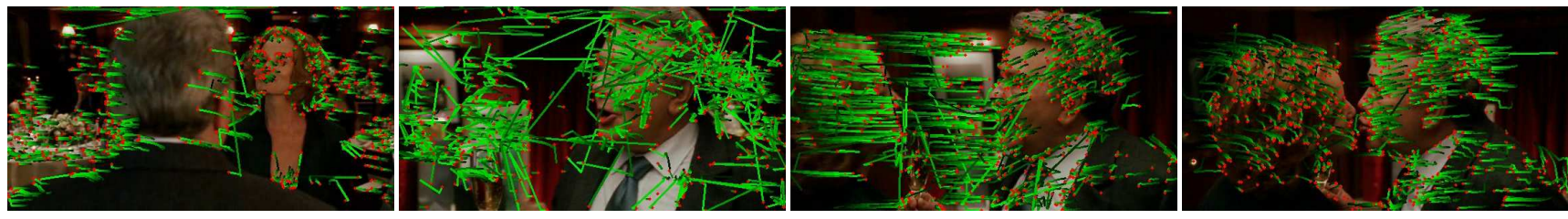

KLT trajectories
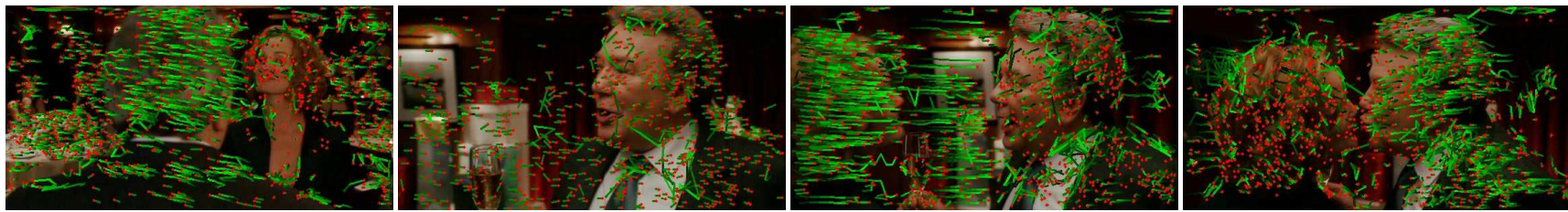

SIFT trajectories
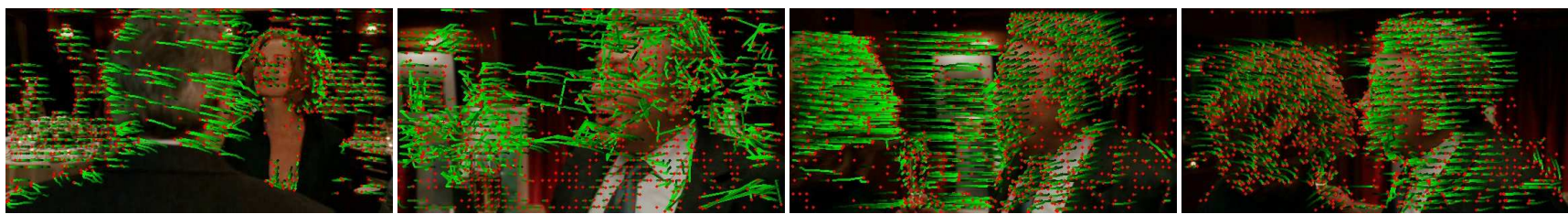

Dense trajectories

Fig. 1 Visualization of KLT, SIFT and dense trajectories for a "kiss" action. Red dots indicate the point positions in the current frame. Compared to KLT trajectories, dense trajectories are more robust to fast irregular motions, in particular at shot boundaries (second column). SIFT trajectories can also handle shot boundaries, but are not able to capture the complex motion patterns accurately.

tectors. In this work, we propose to sample feature points on a dense grid in each frame and track them using a state-ofthe-art dense optical flow algorithm. This allows to improve the quality of the trajectories significantly over sparse tracking techniques, such as the KLT tracker. The resulting trajectories are more robust, in particular in the presence of fast irregular motions (Sand and Teller, 2008; Brox and Malik, 2010; Wang et al, 2011), see Figure 1.

Camera motion is very common in real-world video data such as Hollywood movies and Web videos. To reduce the influence of camera motion on action recognition, we introduce a descriptor based on motion boundaries, initially developed in the context of human detection (Dalal et al, 2006). Motion boundaries are computed by a derivative operation on the optical flow field. Thus, motion due to locally translational camera movement is canceled out and relative motion is captured (see Figure 5). We show that motion boundaries provide a robust descriptor for action recognition that significantly outperforms existing state-of-the-art descriptors.

To evaluate our video description, we perform action classification with a bag-of-features representation and a SVM classifier (Laptev et al, 2008). Spatio-temporal pyramids are used to embed structure information and a multi-channel approach to combine the different features (trajectory shape, HOG, HOF, MBH). We evaluate the improvement of dense trajectories over KLT and SIFT trajectories. Furthermore, we compare different types of descriptors, investigate the impact of various parameters, and study the computational complexity. Experimental results on nine action datasets show a significant improvement over the state of the art.

This paper is organized as follows. Section 2 reviews related work. Section 3 presents our video representation with dense trajectories and motion boundary descriptors. The experimental setup and the datasets are described in sections 4 and 5. Experimental results are given in section 6. Section 7 concludes the paper.

The code for computing dense trajectories and descriptors is available on-line ${ }^{1}$. A preliminary version of this article has appeared in (Wang et al, 2011).

\section{Related work}

There exists a large number of approaches for extracting local spatio-temporal features in videos. Laptev (2005) have introduced spatio-temporal interest points, which are an extension of the Harris detector from image to video. Interest points are local maxima of a cornerness criterion based on the spatio-temporal second-moment matrix at each video point. Dollár et al (2005) have proposed a cornerness function that combines a 2D Gaussian filter in space with a 1D Gabor filter in time. Bregonzio et al (2009) have extended this approach with 2D Gabor filters of different orientations. The spatio-temporal Hessian detector (Willems et al, 2008) relies on the determinant of the spatio-temporal Hessian matrix. Wong and Cipolla (2007) have added global informa-

\footnotetext{
1 http://lear.inrialpes.fr/software
} 


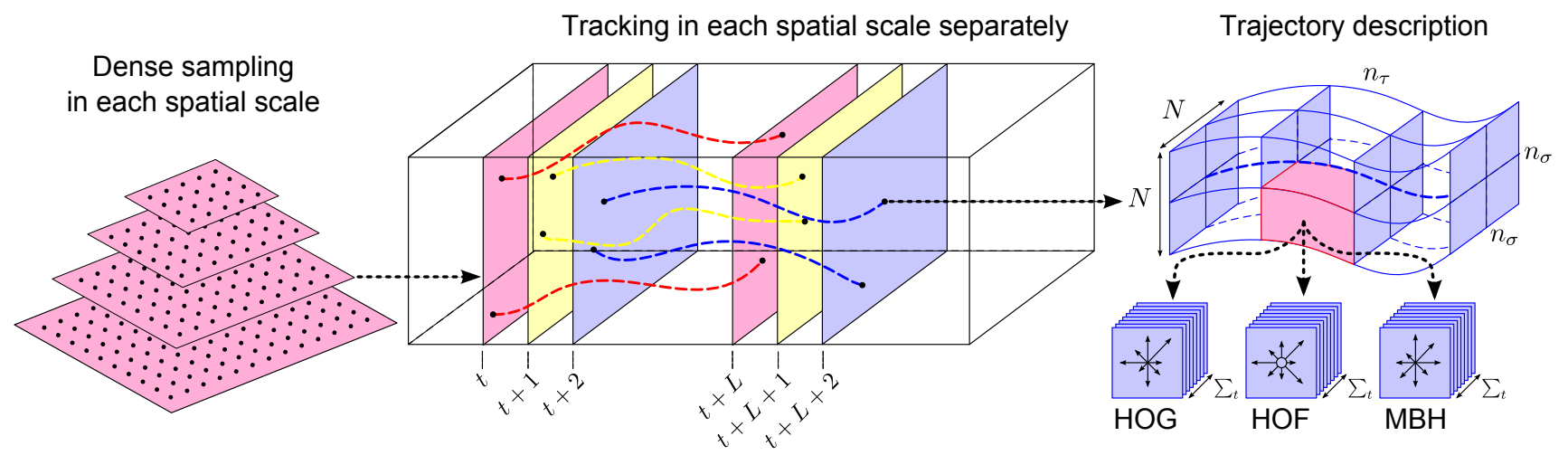

Fig. 2 Illustration of our approach to extract and characterize dense trajectories. Left: Feature points are densely sampled on a grid for each spatial scale. Middle: Tracking is carried out in the corresponding spatial scale for $L$ frames by median filtering in a dense optical flow field. Right: The trajectory shape is represented by relative point coordinates, and the descriptors (HOG, $\mathrm{HOF}, \mathrm{MBH}$ ) are computed along the trajectory in a $N \times N$ pixels neighborhood, which is divided into $n_{\sigma} \times n_{\sigma} \times n_{\tau}$ cells.

tion to the interest point detection by applying non-negative matrix factorization (NNMF) on the entire video sequence. The locations extracted by all these approaches are sparse and detect salient motion patterns.

To describe spatio-temporal points, Schüldt et al (2004) use higher order derivatives (local jets). Dollár et al (2005) rely on descriptors based on normalized brightness, gradient, and optical flow information. Scovanner et al (2007) extend the popular SIFT descriptor (Lowe, 2004) to the spatiotemporal domain, and Kläser et al (2008) introduce the HOG3D descriptor. Willems et al (2008) generalizes the image SURF descriptor (Bay et al, 2006) to the video domain by computing weighted sums of uniformly sampled responses of spatio-temporal Haar wavelets. Yeffet and Wolf (2009) propose Local Trinary Patterns for videos as extension of Local Binary Patterns (LBP) (Ojala et al, 2002). Laptev et al (2008) combine histograms of oriented gradients (HOG) and histograms of optical flow (HOF). Their descriptors show state-of-the-art results in a recent evaluation (Wang et al, 2009).

Spatio-temporal interest points encode video information at a given location in space and time. In contrast, trajectories track a given spatial point over time and, thus, capture motion information. Messing et al (2009) extract feature trajectories by tracking Harris3D interest points (Laptev, 2005) with a KLT tracker (Lucas and Kanade, 1981). Trajectories are represented as sequences of log-polar quantized velocities and used for action classification. Matikainen et al (2009) extract trajectories using a standard KLT tracker, cluster the trajectories, and compute an affine transformation matrix for each cluster center. The elements of the matrix are then used to represent the trajectories. Sun et al (2009) compute trajectories by matching SIFT descriptors between two consecutive frames. They impose a unique-match constraint among the descriptors and discard matches that are too far apart. Actions are described with intra- and inter-trajectory statistics. Sun et al (2010) combine both KLT tracker and SIFT descriptor matching to extract long-duration trajectories. To assure a dense coverage with trajectories, random points are sampled for tracking within the region of existing trajectories. Spatio-temporal statistics of the trajectories are then used to discriminate different actions. Raptis and Soatto (2010) track feature points in regions of interest. They compute tracklet descriptors as concatenation of HOG or HOF descriptors along the trajectories. The final descriptor is employed for action modeling and video analysis. In the experimental section, we compare to KLT and SIFT trajectories as well as to the results of Sun et al (2010) and Messing et al (2009).

Our trajectories differ from previous methods as points are sampled densely and tracked using a dense optical flow field. Dense sampling ensures a good coverage of the video with features, and optical flow improves the quality of trajectories. Dense trajectories have not been employed previously for action recognition. Somewhat related is the approach of Brox and Malik (2010), where long term trajectories are extracted using dense optical flow. In order to segment moving objects, trajectories are clustered using a pairwise distance measure. A similar approach is proposed by Lu et al (2010). The authors use dense optical flow trajectories to extract objects from video. Sand and Teller (2008) investigate long range motion estimation. Videos are represented as a set of particles whose trajectories are computed from variational optical flow.

Previous works also use trajectories at the object level, e.g., for humans or vehicles. Johnson and Hogg (1996) propose to track humans and model the distribution of trajectories in order to identify atypical events. Mean shift is applied to cluster object trajectories based on multiple features in (Anjum and Cavallaro, 2008), and clusters with less trajectories are considered as rare events. Similarly, Jung et al (2008) design a framework for event detection in video 


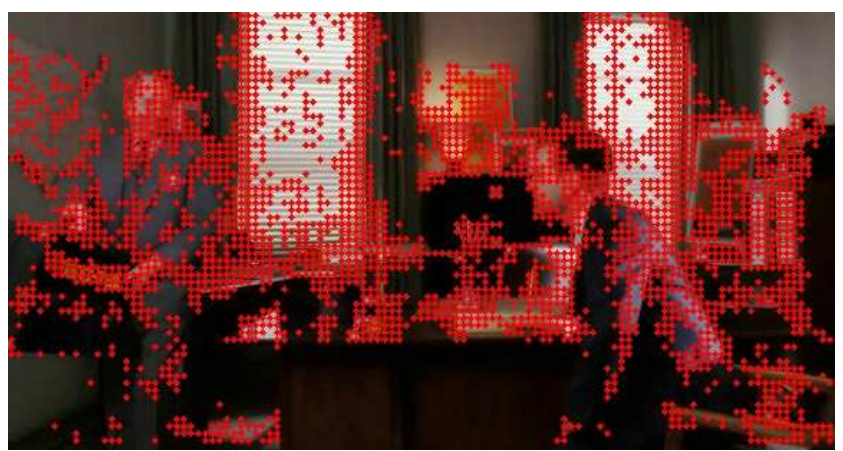

Fig. 3 Visualization of densely sampled feature points after removing points in homogeneous areas using the criterion in Eq. (1). We only show feature points in the first spatial scale. The sampling step size $W$ is 5 pixels, which is the default setting in our experiments.

surveillance based on trajectory clustering of objects and 4-D histograms. Hervieu et al (2008) use Hidden Markov Models to capture the temporal causality of object trajectories for unexpected event detection. Wang et al (2008) propose a nonparametric Bayesian model for trajectory analysis and semantic region modeling in surveillance.

To take into account camera motion, Piriou et al (2006) define global probabilistic motion models for both the dominant image motion (assumed to be due to camera motion) and the residual image motion (related to scene motion) to recognize dynamic video content. In the context of action recognition based on local features, only a few approaches account for camera motion. Uemura et al (2008) segment feature tracks to separate motion characterizing actions from the dominant camera motion. Ikizler-Cinbis and Sclaroff (2010) apply video stabilization by motion compensation to remove camera motion. Recently, Wu et al (2011a) decompose Lagrangian particle trajectories into camera-induced and objectinduced components for videos acquired by a moving camera. In contrast to these approaches, our descriptor is based on motion boundary histograms which remove constant motion and therefore reduce the influence of camera motion.

\section{Dense trajectories}

In this section, we present how to extract dense trajectories and compute trajectory-aligned descriptors. An overview of our approach is shown in Figure 2.

\subsection{Dense sampling}

We first densely sample feature points on a grid spaced by $W$ pixels. Sampling is carried out on each spatial scale separately, see Figure 2(left). This guarantees that feature points equally cover all spatial positions and scales. Experimental results showed that a sampling step size of $W=5$ pixels is dense enough to give good results over all datasets. There

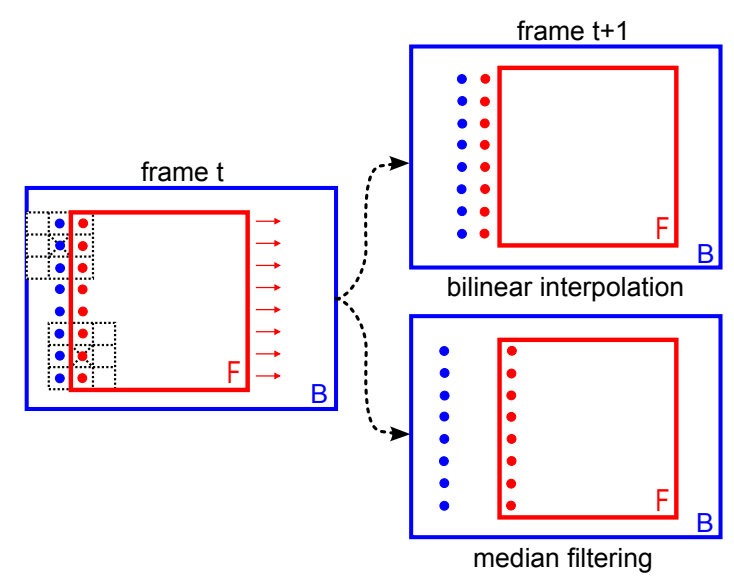

Fig. 4 Comparison of bilinear interpolation and median filtering. The object is moving to the right. Pixels along the motion boundary are indicated by the blue and red dots. The blue dots belonging to the background should stay in place, whereas the red dots should follow the moving foreground object. Using bilinear interpolation, motion boundaries are blurred, and thus foreground and background motion information are confused. Median filtering allows to maintain a sharp motion boundary.

are at most 8 spatial scales in total, depending on the resolution of the video. The spatial scale increases by a factor of $1 / \sqrt{2}$.

Our goal is to track all these sampled points through the video. However, in homogeneous image areas without any structure, it is impossible to track any point. We remove points in these areas. Here, we use the criterion of Shi and Tomasi (1994), that is points on the grid are removed, if the eigenvalues of the auto-correlation matrix are very small. We set a threshold $T$ on the eigenvalues for each frame $I$ as

$T=0.001 \times \max _{i \in I} \min \left(\lambda_{i}^{1}, \lambda_{i}^{2}\right)$,

where $\left(\lambda_{i}^{1}, \lambda_{i}^{2}\right)$ are the eigenvalues of point $i$ in the image $I$. Experimental results showed that a value of 0.001 represents a good compromise between saliency and density of the sampled points. Sampled points of an example frame are illustrated in Figure 3. It can be seen that most points in homogeneous areas have been removed.

\subsection{Trajectories}

Feature points are tracked on each spatial scale separately. For each frame $I_{t}$, its dense optical flow field $\omega_{t}=\left(u_{t}, v_{t}\right)$ is computed w.r.t. the next frame $I_{t+1}$, where $u_{t}$ and $v_{t}$ are the horizontal and vertical components of the optical flow. Given a point $P_{t}=\left(x_{t}, y_{t}\right)$ in frame $I_{t}$, its tracked position in frame $I_{t+1}$ is smoothed by applying a median filter on $\omega_{t}$ :

$P_{t+1}=\left(x_{t+1}, y_{t+1}\right)=\left(x_{t}, y_{t}\right)+\left.\left(M * \omega_{t}\right)\right|_{\left(x_{t}, y_{t}\right)}$,

where $M$ is the median filtering kernel. The size of the median filter kernel $M$ is $3 \times 3$ pixels. As the median filter 


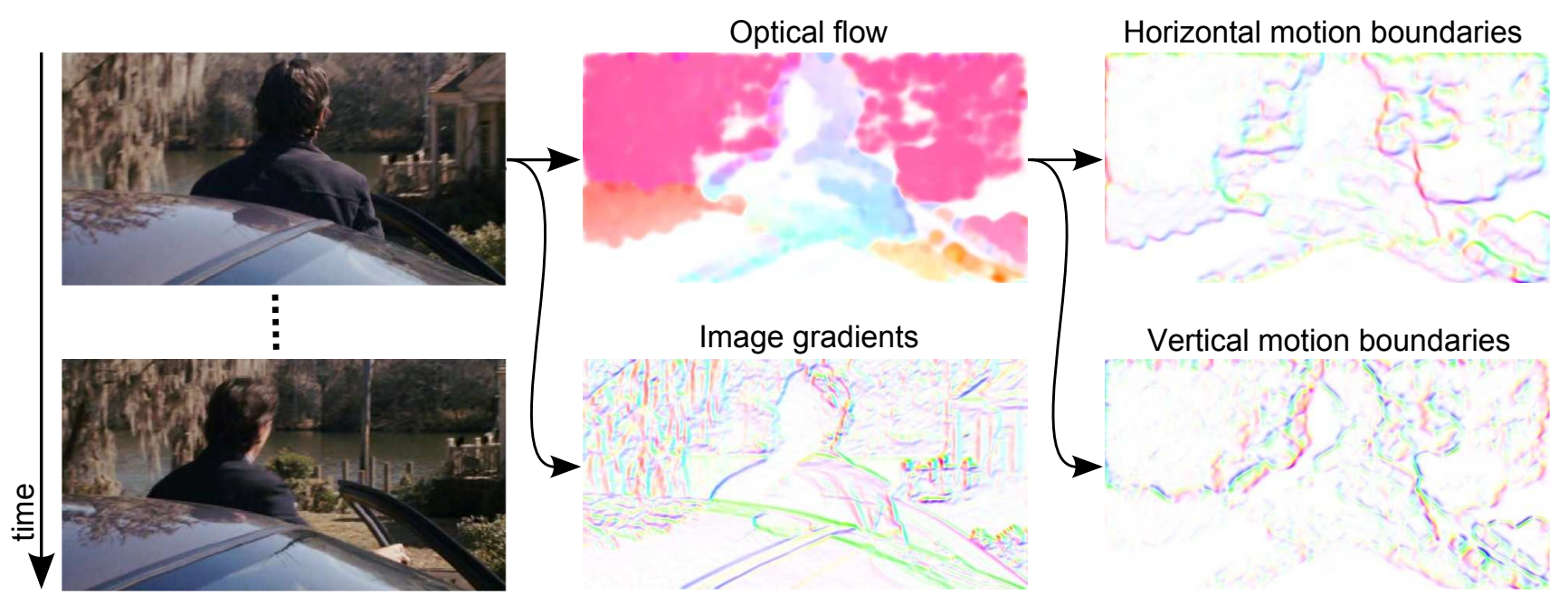

Fig. 5 Illustration of the information captured by HOG, HOF, and MBH descriptors. The camera is moving from right to left, and the person is walking away from the camera. Gradient/flow orientation is indicated by color (hue) and magnitude by saturation. The optical flow (top, middle) shows constant motion in the background, which is due to the camera movements. The motion boundaries (right) encode the relative motion between the person and the background.

is more robust to outliers than bilinear interpolation (as used by Sundaram et al (2010)), it improves trajectories for points at motion boundaries that would otherwise be smoothed out (c.f., Figure 4).

Once the dense optical flow field is computed, points can be tracked very densely without additional cost. Another advantage of the dense optical flow is the smoothness constraints which allow relatively robust tracking of fast and irregular motion patterns, see Figure 1. To extract dense optical flow fields, we use the algorithm by Farnebäck (2003) which embeds a translation motion model between neighborhoods of two consecutive frames. Polynomial expansion is employed to approximate pixel intensities in the neighborhood. We use the implementation from the OpenCV library ${ }^{2}$.

Points of subsequent frames are concatenated to form trajectories: $\left(P_{t}, P_{t+1}, P_{t+2}, \ldots\right)$. As trajectories tend to drift from their initial locations during the tracking process, we limit their length to $L$ frames in order to overcome this problem (c.f., Figure 2 (middle)). For each frame, if no tracked point is found in a $W \times W$ neighborhood, a new point is sampled and added to the tracking process so that a dense coverage of trajectories is ensured. Empirically, we set the trajectory length to $L=15$ frames (c.f., section 6.4).

As static trajectories do not contain motion information, we prune them in a post-processing stage. Trajectories with sudden large displacements, most likely to be erroneous, are also removed. Such trajectories are detected, if the displacement vector between two consecutive frames is larger than $70 \%$ of the overall displacement of the trajectory.

2 http://opencv.willowgarage.com/wiki/
Figure 1 shows the resulting dense trajectories and compares them to KLT and SIFT trajectories. We can observe that dense trajectories are more coherent.

\subsubsection{Trajectory shape descriptor}

The shape of a trajectory encodes local motion patterns. Given a trajectory of length $L$, we describe its shape by a sequence $\left(\Delta P_{t}, \ldots, \Delta P_{t+L-1}\right)$ of displacement vectors $\Delta P_{t}=\left(P_{t+1}-\right.$ $\left.P_{t}\right)=\left(x_{t+1}-x_{t}, y_{t+1}-y_{t}\right)$. The resulting vector is normalized by the sum of displacement vector magnitudes:

$T=\frac{\left(\Delta P_{t}, \ldots, \Delta P_{t+L-1}\right)}{\sum_{j=t}^{t+L-1}\left\|\Delta P_{j}\right\|}$.

In the following, we refer to this vector as trajectory. As we use trajectories with a fixed length of $L=15$ frames, we obtain a 30 dimensional descriptor.

\subsection{Motion and structure descriptors}

Besides the trajectory shape information, we also design descriptors to embed appearance and motion information. Previous local descriptors (Dollár et al, 2005; Kläser et al, 2008; Laptev et al, 2008; Scovanner et al, 2007; Willems et al, 2008) are usually computed in a 3D video volume around interest points. These representations ignore the intrinsic dynamic structures in the video. We compute descriptors within a space-time volume aligned with a trajectory to encode the motion information, Figure 2 (right). The size of the volume is $N \times N$ pixels and $L$ frames long. To embed structure information, the volume is subdivided into a spatio-temporal grid of size $n_{\sigma} \times n_{\sigma} \times n_{\tau}$. We compute a descriptor (e.g., 
$\mathrm{HOG}, \mathrm{HOF}$ or $\mathrm{MBH}$ ) in each cell of the spatio-temporal grid, and the final descriptor is a concatenation of these descriptors. The default parameters for our experiments are $N=32, n_{\sigma}=2, n_{\tau}=3$, which showed to give best performance when cross validating on the training set of Hollywood2. We evaluate the performance of different values for this parameter in section 6.4.

\subsubsection{Gradient and optical flow histograms}

As shown by (Wang et al, 2009), HOG (histograms of oriented gradients) and HOF (histograms of optical flow) descriptors (Laptev et al, 2008) yield excellent results on a variety of datasets in comparison with other state-of-the-art descriptors for action recognition. HOG (Dalal and Triggs, 2005) focuses on static appearance information, whereas HOF captures the local motion information.

We compute the HOG and HOF descriptors along the dense trajectories. For both HOG and HOF, orientations are quantized into 8 bins with full orientation and magnitudes are used for weighting. An additional zero bin is added for HOF (i.e., in total 9 bins) (Laptev et al, 2008). It accounts for pixels whose optical flow magnitudes are lower than a threshold. Both descriptors are normalized with their $L_{2}$ norm. The final descriptor size is 96 for HOG (i.e., $2 \times 2 \times 3 \times 8$ ) and 108 for HOF (i.e., $2 \times 2 \times 3 \times 9$ ). Figure 5 (middle) visualizes the gradient and optical flow information for HOG and HOF.

\subsubsection{Motion boundary histograms}

Optical flow represents the absolute motion between two frames, which contains motion from many sources, i.e., foreground object motion and background camera motion. If camera motion is considered as action motion, it may corrupt the action classification. Various types of camera motion can be observed in realistic videos, e.g., zooming, tilting, rotation, etc. In many cases, camera motion is locally translational and varies smoothly across the image plane.

Dalal et al (2006) proposed the motion boundary histograms (MBH) descriptor for human detection by computing derivatives separately for the horizontal and vertical components of the optical flow. The descriptor encodes the relative motion between pixels, as shown in Figure 5 (right). Since $\mathrm{MBH}$ represents the gradient of the optical flow, locally constant camera motion is removed and information about changes in the flow field (i.e., motion boundaries) is kept. $\mathrm{MBH}$ is more robust to camera motion than optical flow, and thus more discriminative for action recognition.

In this work, we employ $\mathrm{MBH}$ as motion descriptor for trajectories. The MBH descriptor separates optical flow $\omega=$ $(u, v)$ into its horizontal and vertical components. Spatial derivatives are computed for each of them and orientation information is quantized into histograms. The magnitude is used for weighting. We obtain a 8-bin histogram for each component (i.e., MBHx and MBHy). Both histogram vectors are normalized separately with their $L_{2}$ norm. The dimension is 96 (i.e., $2 \times 2 \times 3 \times 8$ ) for both MBHx and MBHy.

Compared to video stabilization (Ikizler-Cinbis and Sclaroff, 2010) and motion compensation (Uemura et al, 2008), this is a simpler way to discount for camera motion. The $\mathrm{MBH}$ descriptor is shown to outperform significantly the HOF descriptor in our experiments, see section 6 .

For both $\mathrm{HOF}$ and $\mathrm{MBH}$ descriptor computation, we reuse the dense optical flow that is already computed to extract dense trajectories. This makes our feature computation process more efficient. A detailed analysis of the complexity is given in section 6.5 .

\section{Experimental setup}

In this section, we first introduce our baseline methods for trajectory extraction. We then detail the bag-of-features representation as used in our experiments and finally present spatio-temporal pyramids.

\subsection{Baseline trajectories}

To quantify the improvement obtained with our dense trajectories, we compare to three baseline trajectories in our experimental results: KLT trajectories (Matikainen et al, 2009; Messing et al, 2009; Sun et al, 2010), SIFT trajectories (Sun et al, 2009, 2010), as well as dense cuboids. Details are presented in the following.

\subsubsection{KLT trajectories}

A standard KLT tracker (Lucas and Kanade, 1981) is employed to construct KLT trajectories. More specifically, about 100 interest points are detected in each frame and are added to the tracker - this is somewhat denser than space-time interest points (Wang et al, 2009). The points are tracked through the video for $L=15$ frames. Then they are removed and replaced by new interest points. This is identical to the procedure used for dense trajectories. We use the same descriptors for KLT trajectories, i.e., the trajectory shape is represented by normalized displacement vectors and HOG, $\mathrm{HOF}$, as well as $\mathrm{MBH}$ descriptors are extracted along the trajectories. We use the OpenCV implementation of the KLT tracker. We also examine the impact of an increasing number of KLT trajectories obtained by decreasing the threshold to extract interest points. 


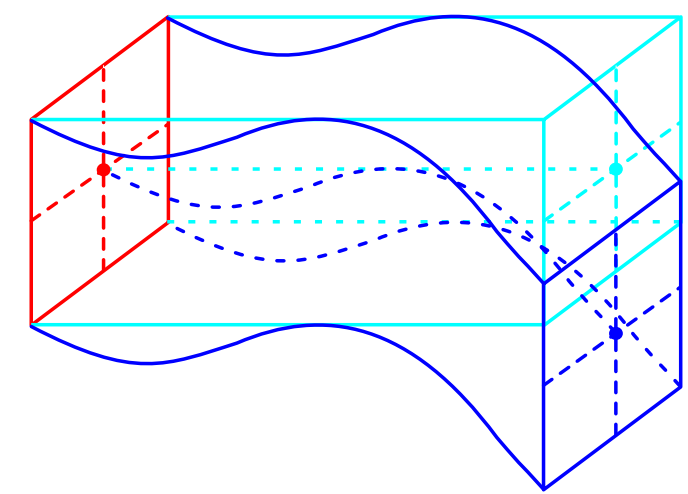

Fig. 6 Illustration of the difference between a dense cuboid and a dense trajectory. Both approaches start at the same position (the red dot), but then continue differently.

\subsubsection{SIFT trajectories}

To extract SIFT trajectories, we first extract SIFT interest points. The best match in the next frame is the point with the smallest Euclidean distance between the SIFT descriptors ${ }^{3}$ within a neighborhood. We set the contrast threshold for the SIFT detector to 0.004 , which is one order lower than the default setting, and makes sure that there are enough SIFT interest points for matching. We further decrease the threshold to extract more SIFT points to obtain additional trajectories and examine the impact on the recognition performance (c.f., Figure 9).

A visualization of SIFT trajectories is represented in Figure 1. Unlike the KLT trajectories, SIFT trajectories are very robust to shot boundaries, i.e., most trajectories crossing the shot boundaries are removed. This is due to the descriptive power of SIFT. However, we observed that SIFT trajectories are not able to model subtle motion changes in videos. This is presumably due to the fact that the descriptor is based on spatial histograms, which are not well localized (Brox and Malik, 2011). Moreover, SIFT interest points are only detected on a set of discrete spatial positions, whereas dense optical flow can provide subpixel accuracy.

\subsubsection{Dense cuboids}

We compare to dense cuboids in order to demonstrate the benefit of descriptors aligned to trajectories. To this end, feature points are sampled in the same way as for dense trajectories. This guarantees that both dense cuboids and dense trajectories have the same number of features at the same positions. The only difference is that feature points are not tracked for dense cuboids. As shown in Figure 6, descriptors are extracted within a straight 3D block. HOG, HOF and $\mathrm{MBH}$ descriptors are computed in this block using the same parameters as for dense trajectories.

\footnotetext{
3 The code of SIFT detector and descriptor is from http:// blogs. oregonstate. edu/hess/code/sift/
}

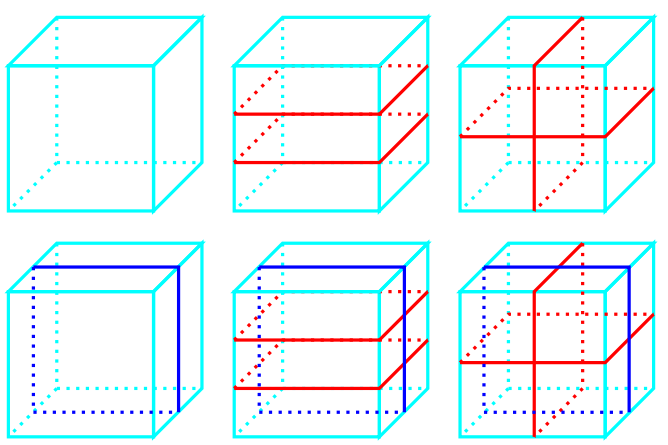

Fig. 7 The spatio-temporal grids used in our experiments: $h 1 \times v 1 \times t 1$, $h 3 \times v 1 \times t 1, h 2 \times v 2 \times t 1, h 1 \times v 1 \times t 2, h 3 \times v 1 \times t 2, h 2 \times v 2 \times t 2$.

\subsection{Bag of features}

We apply the standard bag-of-features approach to evaluate our dense trajectory features as well as the three baseline trajectories. We first construct a codebook for each descriptor (trajectory, $\mathrm{HOG}, \mathrm{HOF}, \mathrm{MBHx}, \mathrm{MBHy})^{4}$ and trajectory type separately. We fix the number of visual words per descriptor to 4000 which has shown to empirically give good results for a wide range of datasets. To limit the complexity, we cluster a subset of 100,000 randomly selected training features using $k$-means. To increase precision, we initialize $k$-means 8 times and keep the result with the lowest error. Descriptors are assigned to their closest vocabulary word using Euclidean distance. The resulting histograms of visual word occurrences are used as video representations.

For classification we use a non-linear SVM with an RBF$\chi^{2}$ kernel (Laptev et al, 2008). Different descriptors are combined in a multi-channel approach (Zhang et al, 2007):

$K\left(x_{i}, x_{j}\right)=\exp \left(-\sum_{c} \frac{1}{A^{c}} D\left(x_{i}^{c}, x_{j}^{c}\right)\right)$,

where $D\left(x_{i}^{c}, x_{j}^{c}\right)$ is the $\chi^{2}$ distance between video $x_{i}$ and $x_{j}$ with respect to the $c$-th channel. $A^{c}$ is the mean value of the $\chi^{2}$ distances between the training samples for the $c$-th channel. In the case of multi-class classification, we use a oneagainst-rest approach and select the class with the highest score.

\subsection{Spatio-temporal pyramids}

We add structure information to the bag of features using spatio-temporal pyramids (Laptev et al, 2008; Ullah et al, 2010), an extension of spatial pyramids for images (Lazebnik et al, 2006). We use in our experiments six different spatio-temporal grids. For the spatial domain we use the entire spatial block $h 1 \times v 1$, a subdivision into three horizontal stripes $h 3 \times v 1$ and a $2 \times 2$ spatial grid $h 2 \times v 2$. For

${ }^{4}$ Note that splitting $\mathrm{MBH}$ into $\mathrm{MBHx}$ and $\mathrm{MBHy}$ results in a slightly better performance. 
the temporal domain we use the entire duration $t 1$ as well as a subdivision into 2 temporal blocks $t 2$. Figure 7 illustrates our six grids, $h 1 \times v 1 \times t 1, h 3 \times v 1 \times t 1, h 2 \times v 2 \times t 1$, $h 1 \times v 1 \times t 2, h 3 \times v 1 \times t 2, h 2 \times v 2 \times t 2$. For each cell of the grid, a separate bag-of-features histogram is computed. The video is, then, represented as concatenation of the cell histograms. We use each grid structure as a separate channel and combined them using Eq. (4). In total, we have 30 different channels (i.e., 6 grid structures $\times 5$ descriptor types) to represent a video.

\section{Datasets}

This section describes the datasets we use and the experimental protocols for these datasets. We extensively evaluate our dense trajectory features on nine action datasets, i.e., KTH, YouTube, Hollywood2, UCF sports, IXMAS, UIUC, Olympic Sports, UCF50 and HMDB51, see Figure 8. These datasets are collected from various sources, e.g., controlled experimental settings, Hollywood movies, Web videos, TV sports, etc. Thus, we investigate the performance of our approach on diverse datasets with different resolutions, viewpoints, illumination changes, occlusion, background clutter, irregular motion, etc. In total, we evaluate over 20,000 video sequences and 200 action classes.

The KTH dataset ${ }^{5}$ (Schüldt et al, 2004) consists of six human action classes: walking, jogging, running, boxing, waving and clapping. Each action is performed several times by 25 subjects. The sequences were recorded in four different scenarios: outdoors, outdoors with scale variation, outdoors with different clothes and indoors. The background is homogeneous and static in most sequences. In total, the data consists of 2,391 video samples. We follow the original experimental setup of the authors, i.e., dividing the samples into test set (9 subjects: 2, 3, 5, 6, 7, 8, 9, 10, and 22) and training set (the remaining 16 subjects). As in the initial paper (Schüldt et al, 2004), we train and evaluate a multi-class classifier and report average accuracy over all classes.

The YouTube dataset ${ }^{6}$ (Liu et al, 2009) contains 11 action categories: basketball shooting, biking/cycling, diving, golf swinging, horse back riding, soccer juggling, swinging, tennis swinging, trampoline jumping, volleyball spiking, and walking with a dog. This dataset is challenging due to large variations in camera motion, object appearance and pose, object scale, viewpoint, cluttered background and illumination conditions. The dataset contains a total of 1,168 sequences. We follow the original setup (Liu et al, 2009), using Leave-One-Out Cross-Validation for a pre-defined set

\footnotetext{
5 http://www.nada.kth.se/cvap/actions/

6 http://www.cs.ucf.edu/ Iiujg/YouTube_ Action_dataset.html
}

of 25 groups. Average accuracy over all classes is reported as the performance measure ${ }^{7}$

The Hollywood2 dataset $^{8}$ (Marszałek et al, 2009) has been collected from 69 different Hollywood movies. There are 12 action classes: answering the phone, driving car, eating, fighting, getting out of car, hand shaking, hugging, kissing, running, sitting down, sitting up, and standing up. In our experiments, we use the clean training set. In total, there are 1,707 video sequences divided into a training set (823 sequences) and a test set (884 sequences). Training and test sequences come from different movies. The performance is evaluated by computing the average precision (AP) for each action class and reporting the mean AP over all classes (mAP) as in Marszałek et al (2009).

The UCF sports dataset ${ }^{9}$ (Rodriguez et al, 2008) contains ten human actions: swinging (on the pommel horse and on the floor), diving, kicking (a ball), weight-lifting, horse-riding, running, skateboarding, swinging (at the high bar), golf swinging and walking. The dataset consists of 150 video samples which show a large intra-class variability. To increase the amount of data samples, we extend the dataset by adding a horizontally flipped version of each sequence to the dataset. Similar to the KTH dataset, we train a multiclass classifier and report average accuracy over all classes. We use the Leave-One-Out setup, i.e., testing on each original sequence while training on all the other sequences together with their flipped versions. Note that the flipped version of the tested sequence is removed from the training set as in (Wang et al, 2009).

IXMAS $^{10}$ (Weinland et al, 2007) is a dataset recorded by cameras from five different viewpoints, as shown in the fifth row of Figure 8. It has 11 action classes, i.e., check watch, cross arms, scratch head, sit down, get up, turn around, walk, wave, punch, kick and pick up. All actions are repeated three times by each of the ten actors and recorded simultaneously from five views, which results in 1,650 sequences in total. We apply Leave-One-Actor-Out Cross-Validation as recommended in the original paper (Weinland et al, 2007), and use samples from all five views for training and testing. Average accuracy over all classes is used as performance measure.

The Olympic Sports dataset ${ }^{11}$ (Niebles et al, 2010) consists of athletes practicing different sports, which are collected from YouTube and annotated using Amazon Mechanical Turk. There are 16 sports actions: high-jump, long-jump, triple-jump, pole-vault, basketball lay-up, bowling, tennis-

\footnotetext{
7 Note that here we use the same dataset as (Liu et al, 2009), whereas in (Wang et al, 2011) we used a different version. This explains the difference in performance on the YouTube dataset.

8 http://lear.inrialpes.fr/data

9 http://server.cs.ucf.edu/ vision/data.html

10 http://4drepository.inrialpes.fr/public/ viewgroup/ 6

11 http://vision.stanford.edu/Datasets/ Olympicsports /
} 

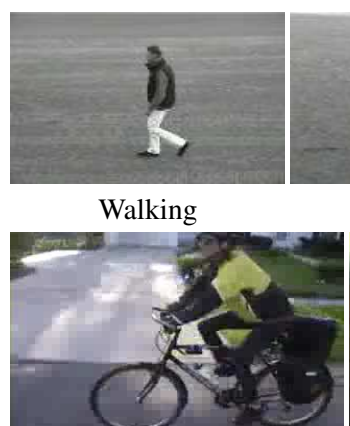

Biking

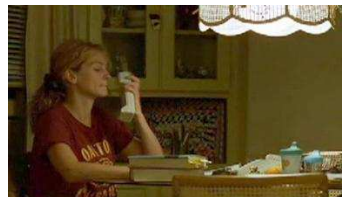

AnswerPhone

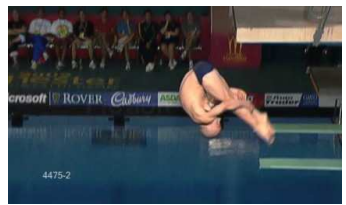

Diving

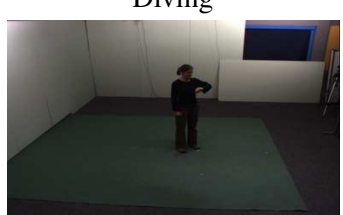

Check-Watch

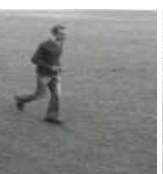

Jogging
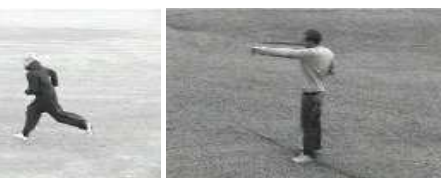

Running

Boxing

Spiking

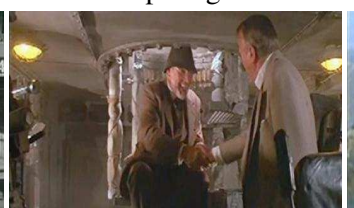

HandShake

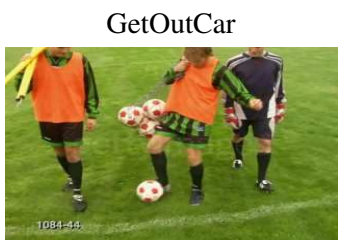

Kicking

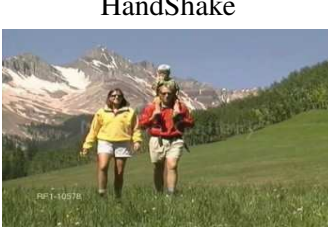

Walking

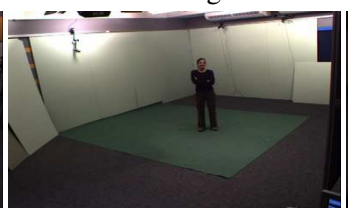

Cross-Arms

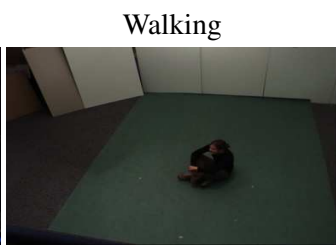

Sit-Down

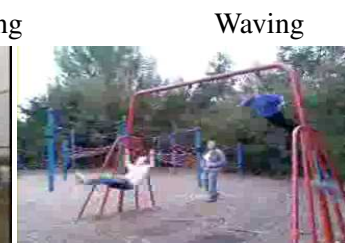

Swinging

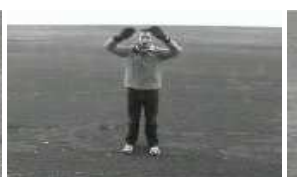

Waving

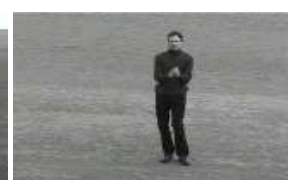

Clapping

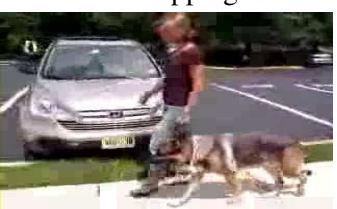

Walking-Dog

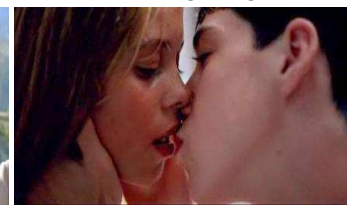

Kiss

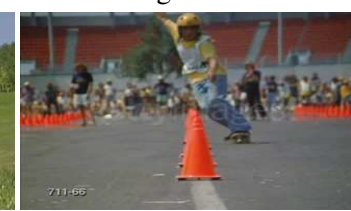

Skateboarding

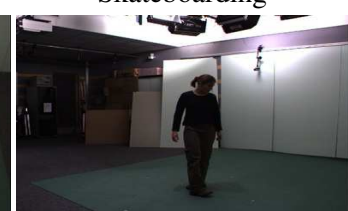

Turn-Around

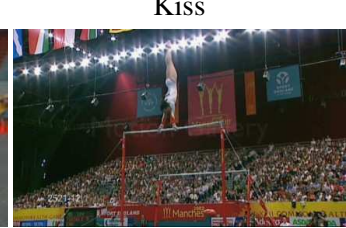

High-Bar-Swinging

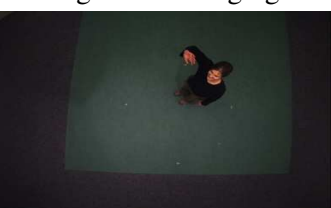

Wave

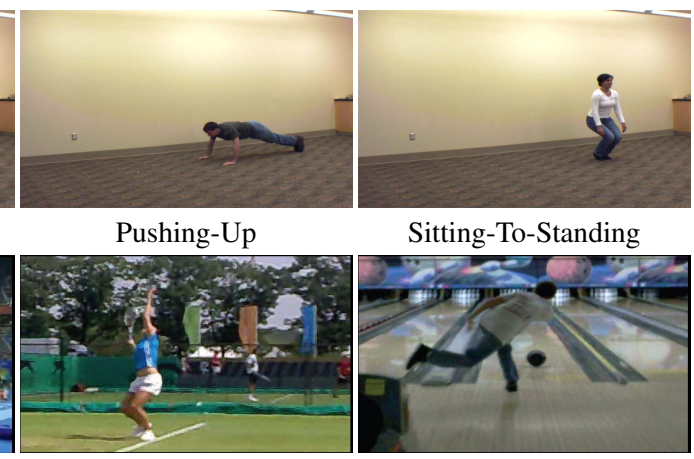

Bowling

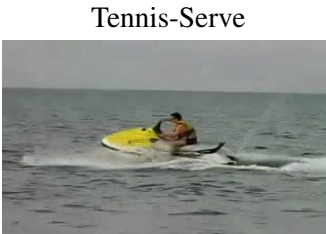

Ski-Jet

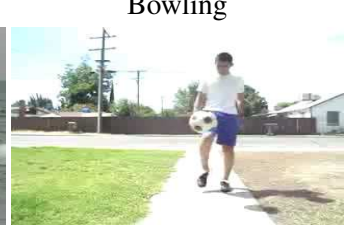

Soccer-Juggling

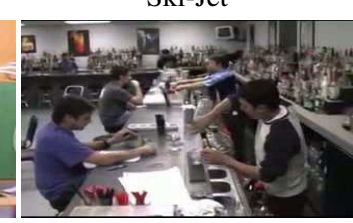

Pour

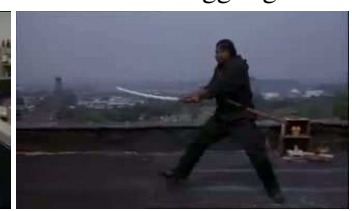

Sword-Exercise

Fig. 8 Sample frames from the nine action recognition datasets used in our experiments. From top to bottom: KTH, YouTube, Hollywood2, UCF sports, IXMAS, UIUC, Olympic Sports, UCF50 and HMDB51. 


\begin{tabular}{|c|c|c|c|c|c|c|c|c|c|c|}
\hline \multicolumn{2}{|c|}{ Datasets } & KTH & YouTube & Hollywood2 & UCF sports & IXMAS & UIUC & Olympic Sports & UCF50 & HMDB51 \\
\hline \multirow{5}{*}{ 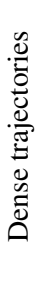 } & Trajectory & $89.8 \%$ & $67.5 \%$ & $47.8 \%$ & $75.4 \%$ & $87.8 \%$ & $98.1 \%$ & $60.5 \%$ & $67.2 \%$ & $28.0 \%$ \\
\hline & HOG & $87.0 \%$ & $72.6 \%$ & $41.2 \%$ & $84.3 \%$ & $82.3 \%$ & $97.9 \%$ & $63.0 \%$ & $68.0 \%$ & $27.9 \%$ \\
\hline & $\mathrm{HOF}$ & $93.3 \%$ & $70.0 \%$ & $50.3 \%$ & $76.8 \%$ & $90.8 \%$ & $97.7 \%$ & $58.7 \%$ & $68.2 \%$ & $31.5 \%$ \\
\hline & $\mathrm{MBH}$ & $95.0 \%$ & $80.6 \%$ & $55.1 \%$ & $84.2 \%$ & $91.8 \%$ & $97.1 \%$ & $71.6 \%$ & $82.2 \%$ & $43.2 \%$ \\
\hline & Combined & $94.2 \%$ & $84.1 \%$ & $58.2 \%$ & $88.0 \%$ & $93.5 \%$ & $98.4 \%$ & $74.1 \%$ & $84.5 \%$ & $46.6 \%$ \\
\hline \multirow{5}{*}{ 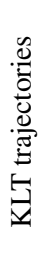 } & Trajectory & $88.4 \%$ & $58.2 \%$ & $46.2 \%$ & $72.8 \%$ & $75.2 \%$ & $96.9 \%$ & $48.1 \%$ & $52.8 \%$ & $22.0 \%$ \\
\hline & HOG & $84.0 \%$ & $71.0 \%$ & $41.0 \%$ & $80.2 \%$ & $74.4 \%$ & $97.3 \%$ & $50.5 \%$ & $57.4 \%$ & $22.2 \%$ \\
\hline & $\mathrm{HOF}$ & $92.4 \%$ & $64.1 \%$ & $48.4 \%$ & $72.7 \%$ & $84.1 \%$ & $97.2 \%$ & $49.2 \%$ & $57.9 \%$ & $23.7 \%$ \\
\hline & $\mathrm{MBH}$ & $93.4 \%$ & $72.9 \%$ & $48.6 \%$ & $78.4 \%$ & $82.5 \%$ & $96.6 \%$ & $59.4 \%$ & $71.1 \%$ & $33.7 \%$ \\
\hline & Combined & $93.4 \%$ & $79.5 \%$ & $54.6 \%$ & $82.1 \%$ & $91.8 \%$ & $98.1 \%$ & $65.5 \%$ & $78.1 \%$ & $40.8 \%$ \\
\hline \multirow{5}{*}{ 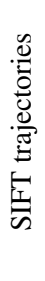 } & Trajectory & $44.6 \%$ & $47.3 \%$ & $39.9 \%$ & $55.7 \%$ & $34.7 \%$ & $87.1 \%$ & $47.4 \%$ & $43.6 \%$ & $17.6 \%$ \\
\hline & HOG & $59.1 \%$ & $59.6 \%$ & $33.3 \%$ & $74.2 \%$ & $45.0 \%$ & $86.2 \%$ & $52.8 \%$ & $53.4 \%$ & $21.2 \%$ \\
\hline & $\mathrm{HOF}$ & $78.8 \%$ & $55.4 \%$ & $38.7 \%$ & $69.9 \%$ & $63.0 \%$ & $91.4 \%$ & $45.0 \%$ & $52.9 \%$ & $22.4 \%$ \\
\hline & $\mathrm{MBH}$ & $79.5 \%$ & $64.5 \%$ & $40.6 \%$ & $72.1 \%$ & $58.9 \%$ & $90.3 \%$ & $54.5 \%$ & $62.7 \%$ & $26.3 \%$ \\
\hline & Combined & $84.9 \%$ & $73.2 \%$ & $45.4 \%$ & $77.9 \%$ & $67.9 \%$ & $90.8 \%$ & $61.5 \%$ & $71.8 \%$ & $32.5 \%$ \\
\hline \multirow{4}{*}{ 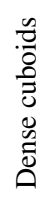 } & HOG & $77.0 \%$ & $70.3 \%$ & $43.3 \%$ & $80.2 \%$ & $79.4 \%$ & $96.5 \%$ & $62.7 \%$ & $64.4 \%$ & $25.2 \%$ \\
\hline & $\mathrm{HOF}$ & $90.5 \%$ & $68.3 \%$ & $48.0 \%$ & $77.8 \%$ & $90.3 \%$ & $97.1 \%$ & $57.3 \%$ & $65.9 \%$ & $29.4 \%$ \\
\hline & $\mathrm{MBH}$ & 93.9\% & $78.4 \%$ & $52.1 \%$ & $83.2 \%$ & $91.2 \%$ & $97.6 \%$ & $66.6 \%$ & $78.3 \%$ & $40.9 \%$ \\
\hline & Combined & $93.1 \%$ & $81.4 \%$ & $55.2 \%$ & $85.5 \%$ & $92.8 \%$ & $97.3 \%$ & $69.3 \%$ & $80.2 \%$ & $43.1 \%$ \\
\hline
\end{tabular}

Table 1 Comparison of different descriptors and methods for extracting trajectories on nine datasets. We report mean average precision over all classes (mAP) for Hollywood2 and Olympic Sports, average accuracy over all classes for the other seven datasets. The three best results for each dataset are in bold.

serve, platform, discus, hammer, javelin, shot-put, springboard, snatch, clean-jerk and vault, represented by a total of 783 video sequences. We use 649 sequences for training and 134 sequences for testing as recommended by the authors. Like UCF sports, this dataset has rich scene context information, which is very helpful for recognizing sports actions. We report mean average precision over all classes (mAP) as in (Niebles et al, 2010).

The UIUC dataset ${ }^{12}$ (Tran and Sorokin, 2008) is recorded in a controlled experimental setting with clean background and fixed cameras. There are 14 action classes, i.e., walking, running, jumping, waving, jumping jacks, clapping, jump from situp, raise one hand, stretching out, turning, sitting to standing, crawling, pushing up and standing to sitting. In total, it contains 532 videos performed by 8 persons. We use Leave-One-Person-Out Cross-Validation following the original paper, and report average accuracy over all classes as performance measure.

\footnotetext{
12 http://vision.cs.uiuc.edu/projects/ activity/
}

The UCF50 dataset ${ }^{13}$ (Reddy and Shah, 2012) has 50 action categories, consisting of real-world videos taken from the YouTube website. This dataset can be considered as an extension of the YouTube dataset. The actions range from general sports to daily life exercises. For all 50 categories, the videos are split into 25 groups. For each group, there are at least 4 action clips. In total, there are 6,618 video clips. The video clips in the same group may share some common features, such as the same person, similar background or similar viewpoint. We apply the same Leave-One-GroupOut Cross-Validation as for the YouTube dataset and report average accuracy over all classes.

The HMDB51 dataset ${ }^{14}$ (Kuehne et al, 2011) is collected from a variety of sources ranging from digitized movies to YouTube videos. It contains simple facial actions, general body movements and human interactions. In total, there are

\footnotetext{
13 http://server.cs.ucf.edu/ vision/data/ $\mathrm{UCF} 50$. rar

14 http://serre-lab.clps.brown.edu/resources/ HMDB /
} 


\begin{tabular}{|l||cc|c|}
\hline Datasets & MBHx & MBHy & MBH \\
\hline \hline KTH & $93.8 \%$ & $94.2 \%$ & $95.0 \%$ \\
YouTube & $76.1 \%$ & $76.0 \%$ & $80.6 \%$ \\
Hollywood2 & $48.5 \%$ & $52.9 \%$ & $55.1 \%$ \\
UCF sports & $83.8 \%$ & $82.9 \%$ & $84.2 \%$ \\
IXMAS & $86.5 \%$ & $89.3 \%$ & $91.8 \%$ \\
UIUC & $95.6 \%$ & $95.6 \%$ & $97.1 \%$ \\
Olympic Sports & $67.4 \%$ & $67.7 \%$ & $71.6 \%$ \\
UCF50 & $75.0 \%$ & $77.9 \%$ & $82.2 \%$ \\
HMDB51 & $32.2 \%$ & $38.8 \%$ & $43.2 \%$ \\
\hline
\end{tabular}

Table 2 Results of MBHx, MBHy and MBH descriptors for dense trajectories on all nine datasets. MBHx and MBHy are combined using the multi-channel approach.

51 action categories and 6,766 video sequences. We follow the original protocol using three train-test splits (Kuehne et al, 2011). For every class and split, there are 70 videos for training and 30 videos for testing. We report average accuracy over the three splits as performance measure. Note that the dataset includes both the original videos and their stabilized version. With the exception of Table 3, we report results for the original videos.

\section{Experimental results}

This section evaluates our dense trajectories and motionboundary descriptors on nine datasets. We first discuss the performance of different descriptors for dense trajectories in section 6.1. In section 6.2, we compare dense trajectories with three baselines, i.e., KLT trajectories, SIFT trajectories and dense cuboids. Section 6.3 presents the results of dense trajectories using different optical flow algorithms. The parameters of our approach are evaluated in section 6.4 and the computational complexity is analyzed in section 6.5. Finally, we compare to state-of-the-art results in section 6.6.

We use the same default parameters on all datasets. Unless stated otherwise, we set $N=32, n_{\sigma}=2, n_{\tau}=3$, and fix the trajectory length $L$ to 15 frames. The sampling step size is $W=5$ pixels.

\subsection{Comparison of different descriptors}

The different descriptors are compared in Table 1. We report the performance of each individual descriptor (i.e., trajectory, $\mathrm{HOG}, \mathrm{HOF}$ and $\mathrm{MBH}$ ), and the overall combination using the multi-channel approach (c.f., Eq. (4)). The results for $\mathrm{MBH}$ are obtained by combining MBHx and MBHy channels.

\begin{tabular}{|l||cc|}
\hline Datasets & original & stabilized \\
\hline \hline Trajectory & $28.0 \%$ & $34.0 \%$ \\
HOG & $27.9 \%$ & $30.1 \%$ \\
HOF & $31.5 \%$ & $39.5 \%$ \\
MBH & $43.2 \%$ & $44.9 \%$ \\
\hline Combined & $46.6 \%$ & $50.8 \%$ \\
\hline
\end{tabular}

Table 3 Descriptor comparison on HMDB51 for the original videos and their stabilized version provided by the authors (Kuehne et al, 2011).

The trajectory descriptor gives surprisingly good results by itself, $(89.8 \%$ on KTH, $87.8 \%$ on IXMAS, $98.1 \%$ on UIUC and $47.8 \%$ on Hollywood2). Trajectory shape information is able to outperform HOG on KTH, IXMAS, UIUC and Hollywood2. As the first three datasets all have clean background, tracking is much easier and thus carries more discriminative information. HOG does not perform well on Hollywood 2 as complex cluttered background degrades its discriminative power.

One may expect the HOF descriptor to outperform HOG as motion seems intuitively more discriminative than static appearance for action recognition. However, HOG reports better results on three datasets: YouTube, UCF sports and Olympic Sports. In fact, all three datasets contain a significant amount of sports actions. UCF sports and Olympic Sports are both by definition sports datasets, whereas a large part of actions in YouTube are sports-related, e.g., basketball shooting, biking, golf swinging, etc. Spatial context is very informative for sports actions as they usually involve specific equipments and particular environments. HOG is designed to encode this static context information and, thus, is more suitable for sports actions. Additionally, the performance of HOF can be corrupted by camera motion for these datasets.

MBH consistently outperforms the other descriptors on all the datasets except UIUC. The improvement over HOF is most significant on real-world datasets. For instance, $\mathrm{MBH}$ is $14 \%$ better than HOF on UCF50. We can also observe an improvement of over $10 \%$ on Olympic Sports, YouTube and HMDB51. This indicates that suppressing camera motion is advantageous for videos from real-world datasets as they are often collected by hand-held cameras, e.g., YouTube and UCF50. For datasets with fixed cameras, e.g., IXMAS and UIUC, the results for $\mathrm{MBH}$ and $\mathrm{HOF}$ are very similar.

We show the results for MBHx and MBHy separately in Table 2. Generally, MBHx and MBHy show similar performance. Interestingly, MBHy outperforms MBHx by $4.5 \%$ on Hollywood2. A possible explanation is that this dataset contains several actions, such as sit up, sit down and stand 


\begin{tabular}{|l||c|c|c||c|c|c|c|}
\hline \multirow{2}{*}{ Datasets } & \multicolumn{2}{||}{ Descriptors of (Laptev et al, 2008) } & \multicolumn{4}{c|}{ Our descriptors } \\
\cline { 2 - 8 } & HOG & HOF & Combined & HOG & HOF & MBH & Combined \\
\hline \hline YouTube & $61.7 \%$ & $61.3 \%$ & $69.2 \%$ & $62.5 \%$ & $62.1 \%$ & $70.9 \%$ & $76.2 \%$ \\
Hollywood2 & $41.2 \%$ & $43.6 \%$ & $47.7 \%$ & $40.4 \%$ & $44.9 \%$ & $47.2 \%$ & $51.9 \%$ \\
\hline
\end{tabular}

Table 4 Descriptor comparison on YouTube and Hollywood2. Descriptors are computed at space-time interest point locations (Laptev et al, 2008).

up, which are dominant in the vertical direction. Similar observations hold for IXMAS, UCF50 and HMDB51.

In general, the combination of all the descriptors improves the final performance significantly, see Table 1. Results are $3 \%$ better than the single best descriptor on YouTube, Hollywood2, UCF sports and HMDB51. For datasets from controlled settings, the improvement is less significant. On $\mathrm{KTH}, \mathrm{MBH}$ alone is even slightly better (i.e., 95.0\%) than combining all the descriptors together.

We investigate the impact of video stabilization on the video description for HMDB51, see Table 3. We can observe that the performance of the trajectory descriptor improves by $6 \%$ as irrelevant trajectories due to camera motion are largely removed in stabilized videos. The improvement for HOF is even more significant, i.e., $8 \%$. This can be explained by its lack of robustness to camera motion. The performance of $\mathrm{MBH}$ is similar in both cases, which demonstrates its robustness to camera motion. The HOG descriptor improves only marginally, as it mainly represents static appearance.

We also compare descriptors computed at space-time interest points extracted with the Harris3D detector (Laptev, 2005). Table 4 compares the HOG and HOF descriptors of Lap et al (2008) to our implementation of HOG and HOF as well as MBH. For our descriptors, we take the positions of Harris $3 \mathrm{D}$ interest points, and then compute descriptors in the 3D patches around these positions with the same parameters as for the dense trajectories, i.e., the size of the 3D patch is $32 \times 32$ pixels and 15 frames. The performance of HOG and HOF descriptors for both methods are comparable as to be expected. MBH outperforms all other descriptors by a large margin and improves significantly over HOF.

\subsection{Comparison to baseline trajectories}

We use the default parameters for characterizing the baselines trajectories, e.g., $N=32, n_{\sigma}=2, n_{\tau}=3$, and a trajectory length $L$ of 15 frames for KLT and SIFT trajectories. The temporal length of dense cuboids is also 15 frames, with the same initial points as for the dense trajectories. Results are given in Table 1.

We first compare the performance of the trajectory descriptor for KLT, SIFT and dense trajectories. On all nine

\begin{tabular}{|l||c|c|c|}
\hline Dataset & Trajectory & Sun et al (2010) & Messing et al (2009) \\
\hline KTH & $89.8 \%$ & $86.8 \%$ & $74.0 \%$ \\
\hline
\end{tabular}

Table 5 Comparing the performance of trajectory descriptor obtained by different methods on the KTH dataset, as reported in the respective papers. Trajectory refers to our trajectory descriptor obtained for dense trajectories.

datasets, the trajectory descriptor obtained with dense trajectories report the best results. The largest improvement over KLT trajectories is $14.4 \%$ on UCF50. On YouTube, IXMAS and Olympic Sports, we can observe an improvement of over $9 \%$. This indicates that our dense trajectories capture better the dynamics in videos. SIFT trajectories perform the worst among the three trajectories, especially on datasets with clean background, e.g., KTH and IXMAS. This can be explained by the fact that SIFT interest points are rather sparse on these datasets and result in non-smooth trajectories.

Table 5 compares the performance of our trajectory shape descriptor with Sun et al (2010) and Messing et al (2009) on the KTH dataset ${ }^{15}$. We can observe that our trajectory deevscriptor (i.e., normalized point coordinates) improves over these two trajectory based methods. This may be due to the quality of our trajectories, i.e., tracking with dense optical flow significantly improves the quality of trajectories. Furthermore, our dense sampling strategy guarantees that features points are distributed equally over all spatial scales and positions, which could explain the significant difference with Messing et al (2009).

The improvement of other descriptors is also substantial as shown in Table 1. The HOF descriptor for dense trajectories is $9.5 \%$ better than HOF computed on KLT trajectories on Olympic Sports, whereas $\mathrm{MBH}$ for dense trajectories outperforms KLT trajectories by $11.1 \%$ on UCF50. In comparison with SIFT trajectories, the performance gain using dense trajectories is even larger. Overall this suggests that the quality of trajectories has a strong influence on the performance of trajectory-aligned descriptors.

We evaluate the difference of descriptors aligned to trajectories in comparison to dense spatio-temporal cuboids. Typically, the improvement of dense trajectories is around

15 Note that we only consider the performance of the trajectory itself. Other information, such as gradient or optical flow, is not included. 


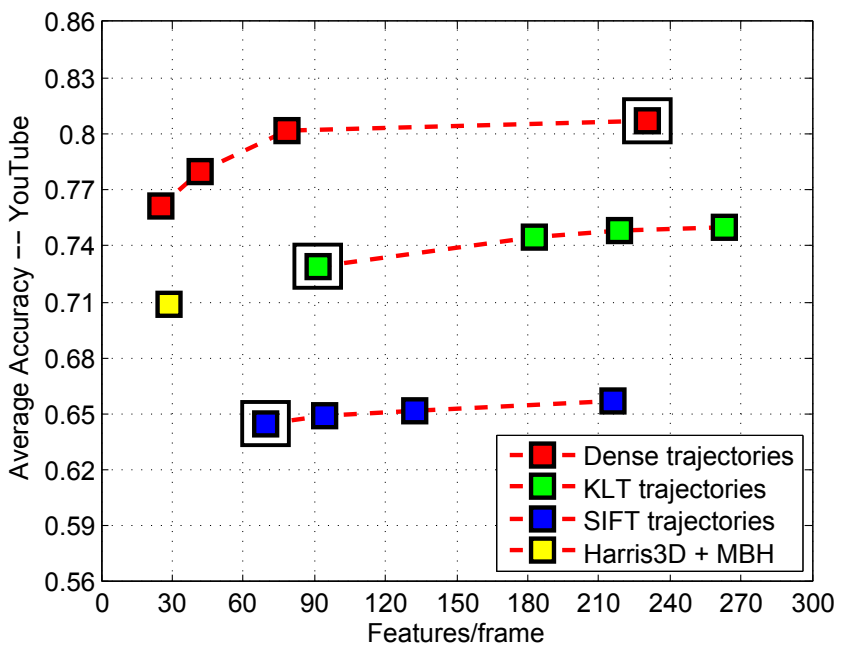

(a) YouTube - MBH

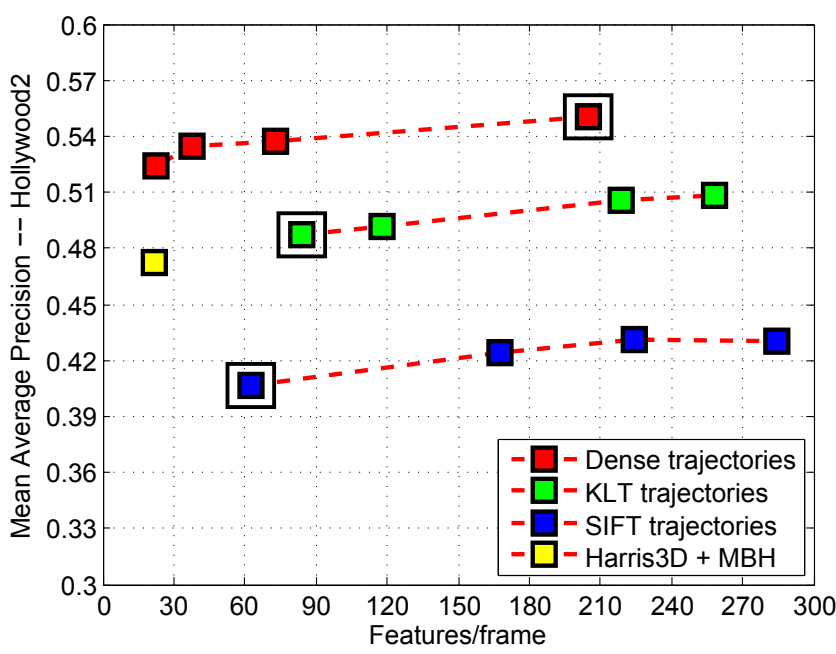

(c) Hollywood2 - MBH

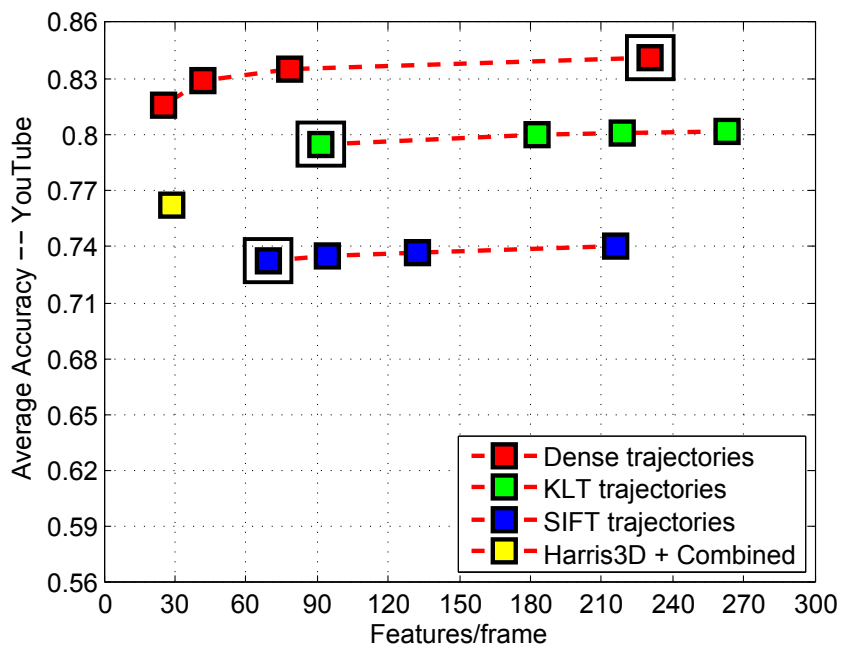

(b) YouTube - Combined

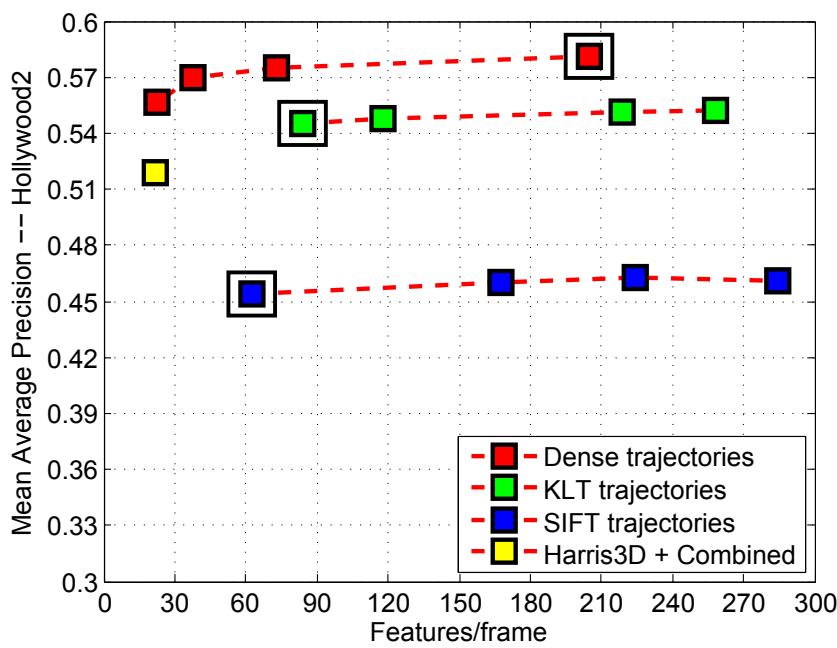

(d) Hollywood2 - Combined

Fig. 9 Performance of dense, KLT and SIFT trajectories for a varying number of features per frame. On the left the results for the MBH descriptor and on the right for a combination of trajectory, HOG, HOF and MBH.

$3 \%$ for a single descriptor. The largest improvement is $10 \%$ for the HOG descriptor on KTH. In a few cases, the descriptors using dense cuboids show slightly better results than those for dense trajectories, e.g., HOF on UCF Sports (1\%). In total, descriptors aligned with trajectories are superior to those computed for straight 3D blocks.

The results for combined descriptors on all trajectory types and dense cuboids show that dense trajectories yield overall the best performance on all nine datasets. The improvement is over $3 \%$ on Hollywood2, Olympic Sports, UCF50 and HMDB51. Interestingly, dense representations (i.e., dense trajectories and dense cuboids) consistently outperform sparse representations (i.e., KLT trajectories and SIFT trajectories) on YouTube, UCF sports, Olympic Sports, UCF50 and HMDB This shows that a high number of features improve the results, especially for sports-related datasets where context information can play an important role. For example, dense trajectories improve by $8.6 \%$ on Olympic Sports over KLT trajectories. However, for datasets collected in controlled experimental settings, e.g., KTH, IXMAS and UIUC, the performance gain is less significant.

We also evaluate the impact of the feature density on the performance of KLT, SIFT and dense trajectories, see Figure 9. The results obtained with the standard parameters and the corresponding average number of features per frame are indicated by a black rectangle. For KLT and SIFT trajectories we gradually decrease the thresholds for extracting features to increase their numbers per frame. For dense trajectories we vary the sampling stride $W$ (i.e., 5, 8, 11, 14 pixels) to obtain fewer feature points. We also include the 51results for Harris3D and our descriptors from Table 4.

We can observe that increasing the number of features for KLT and SIFT increases the performance slightly and then saturates. The performance gain between the default 


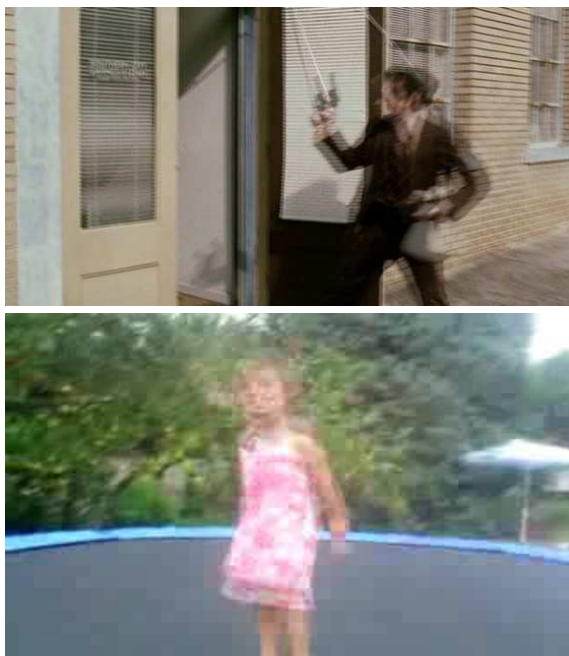

Overlaid image

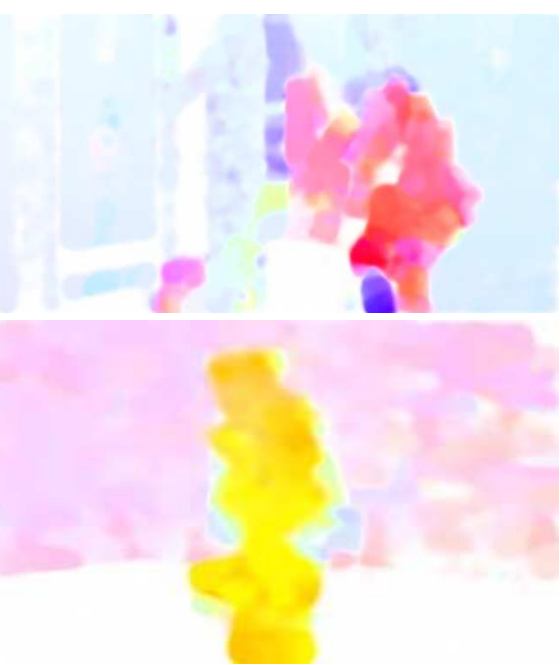

Farnebäck (2003)

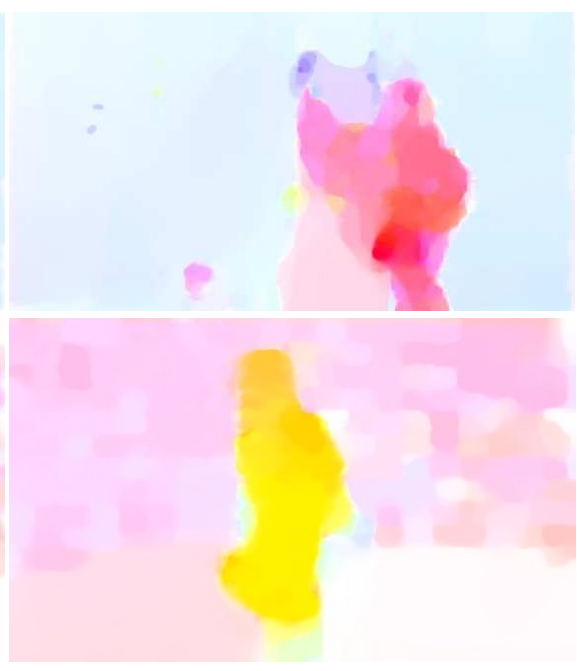

Brox and Malik (2011)

Fig. 10 Examples from Hollywood2 (top) and YouTube (bottom). The left column shows the overlaid image of two consecutive frames. The middle and right columns are the visualization of two optical flow methods Farnebäck (2003) and Brox and Malik (2011).

\begin{tabular}{|l||c|c|c|c|c||c|c|c|c|c|}
\hline \multicolumn{1}{|c||}{ Methods } & \multicolumn{4}{|c||}{ YouTube } & \multicolumn{4}{c|}{ Hollywood2 } \\
\cline { 2 - 11 } & Trajectory & HOG & HOF & MBH & Combined & Trajectory & HOG & HOF & MBH & Combined \\
\hline \hline Farnebäck (2003) & $67.5 \%$ & $72.6 \%$ & $70.0 \%$ & $80.6 \%$ & $84.1 \%$ & $47.8 \%$ & $41.2 \%$ & $50.3 \%$ & $55.1 \%$ & $58.2 \%$ \\
Brox and Malik (2011) & $64.5 \%$ & $71.9 \%$ & $65.7 \%$ & $83.6 \%$ & $84.9 \%$ & $43.7 \%$ & $40.8 \%$ & $46.1 \%$ & $54.3 \%$ & $57.2 \%$ \\
\hline
\end{tabular}

Table 6 Comparing the optical flow algorithms of Farnebäck (2003) and Brox and Malik (2011) for extracting our dense trajectories. Results are reported on the YouTube and Hollywood2 datasets.

parameters and the parameters for which the feature number is comparable to the standard dense trajectories is around $1 \%$ for both KLT and SIFT trajectories. For a comparable number of features, dense trajectories outperform KLT trajectories by around $5 \%$ (3\%) for MBH (combined) results. The improvement over SIFT trajectories is around $12 \%(10 \%)$.

\subsection{Comparison of different optical flow algorithms}

Our implementation of dense trajectories uses the optical flow algorithm of Farnebäck (2003), which represents a good compromise between speed and accuracy. In this section, we compare to a state-of-the-art optical flow algorithm, the large displacement optical flow (LDOF) from Brox and Malik (2011). We replace Farnebäck's optical flow with LDOF, and keep everything else identical. To extract LDOF we use the binary code from the author's website ${ }^{16}$ with the default parameters.

The results are given in Table 6. Surprisingly, the overall performance of the two optical flow algorithms is similar. The result of the MBH descriptor from LDOF is somewhat

\footnotetext{
16 http://lmb.informatik.uni-freiburg.de/ resources/binaries/pami2010Linux64.zip
}

better for the YouTube dataset and slightly worse for Hollywood2. One possible explanation is that we use the default parameters for LDOF, which may not be optimal for realistic Hollywood movies. Another possible explanation is that Farnebäck (2003) estimates optical flow locally and captures fine details in textured areas, whereas LDOF includes global smoothness constraints and preserves motion boundaries, see figure 10. Please note that LDOF is computationally expensive; its run time is significantly higher than that of Farnebäck (2003).

\subsection{Evaluation of trajectory parameters}

In this section, we evaluate the impact of the parameters on dense trajectories. We report results for Hollywood 2 and YouTube. We study the impact of trajectory length, sampling step size, neighborhood size, cell grid structure, spatial scale number and refresh rate (the frame rate for which we sample feature points in time). We carry out the evaluation for one parameter at a time, and fix the other parameters to the default values, i.e., trajectory length $L=15$, sampling step size $W=5$, neighborhood size $N=32$, cell grid structure $n_{\sigma}=2, n_{\tau}=3$, the number of spatial scale $S=8$ and refresh rate $R=1$. 


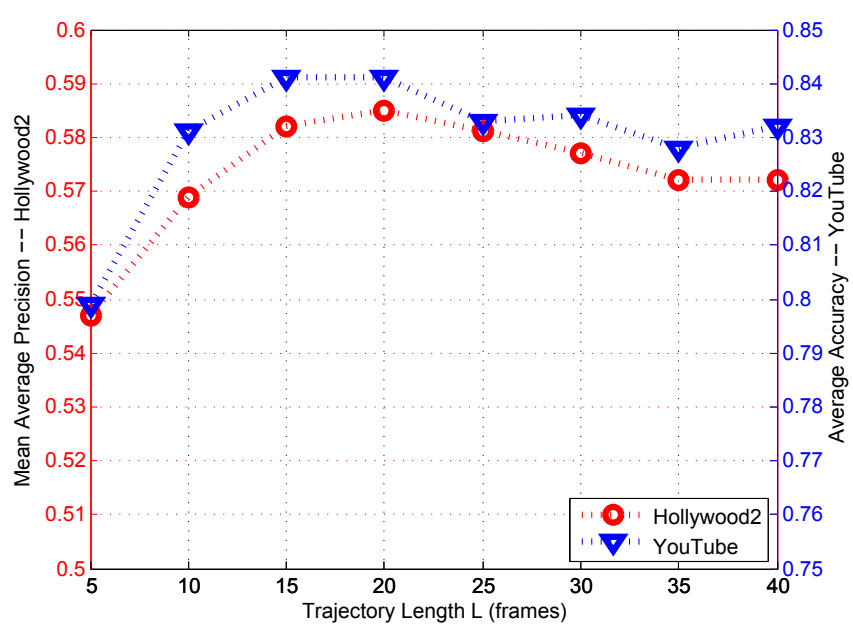

(a)

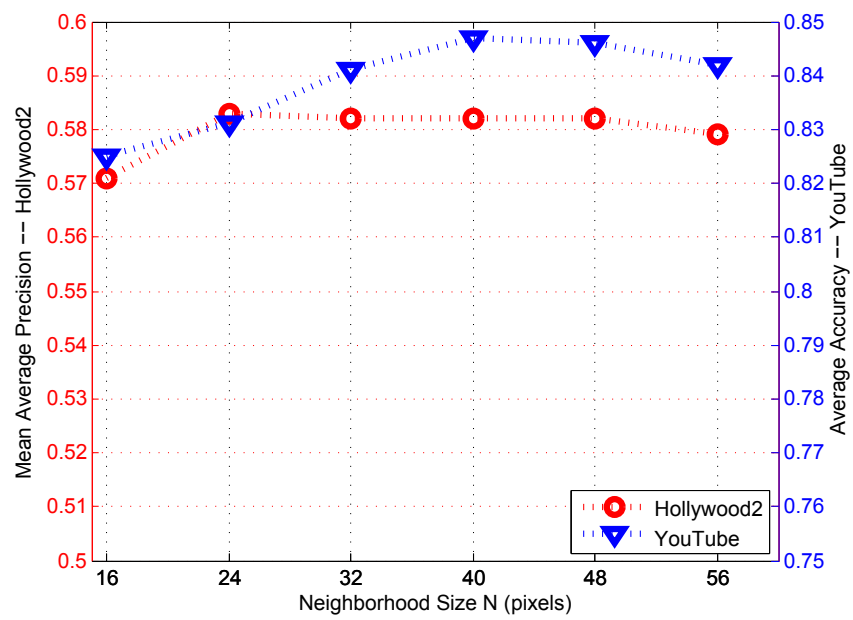

(c)

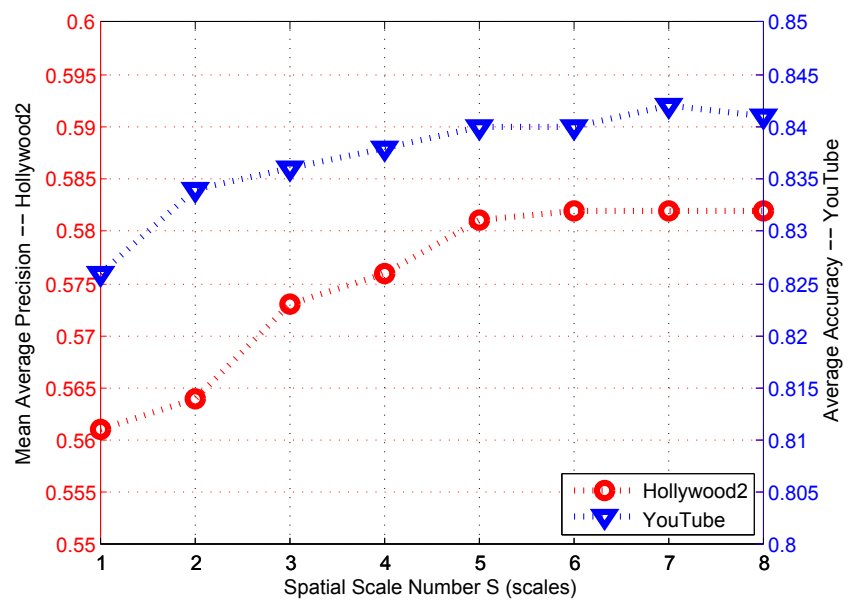

(e)

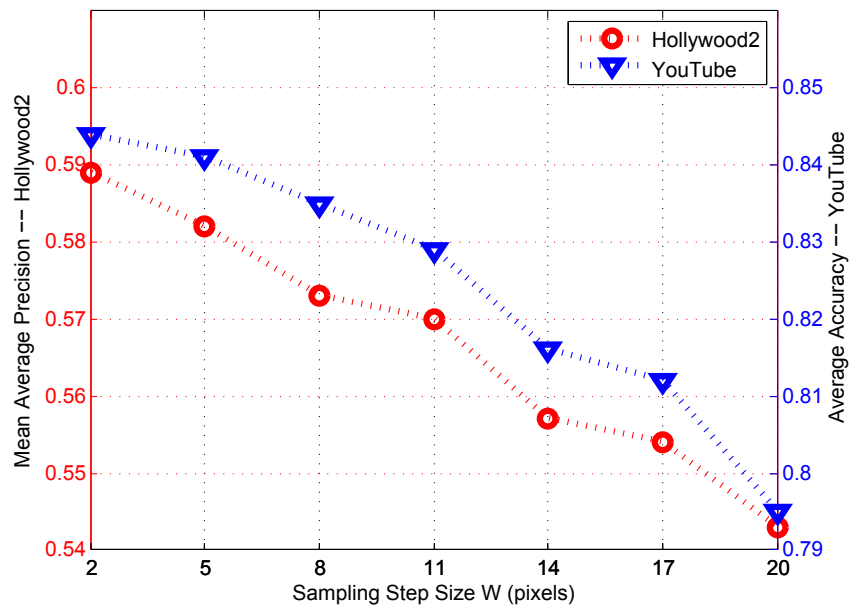

(b)

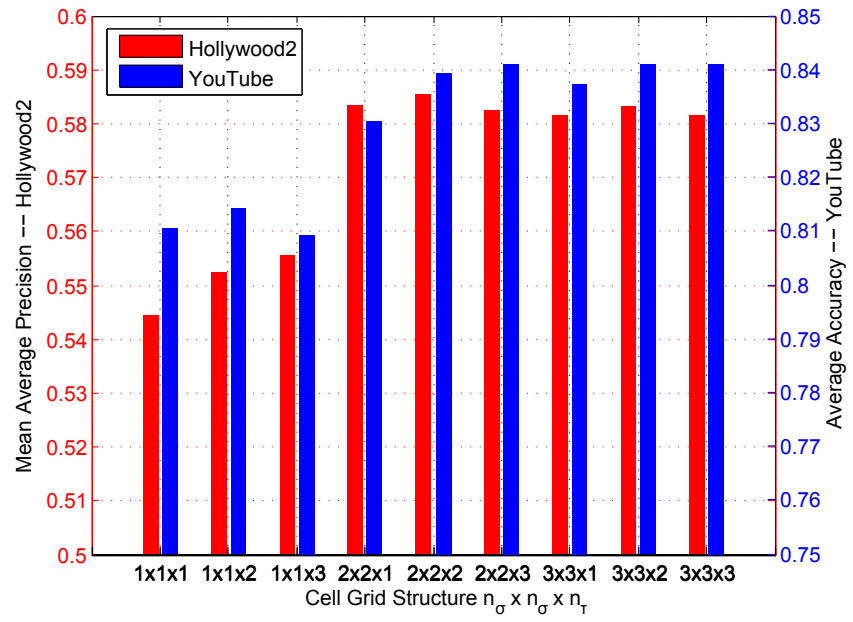

(d)

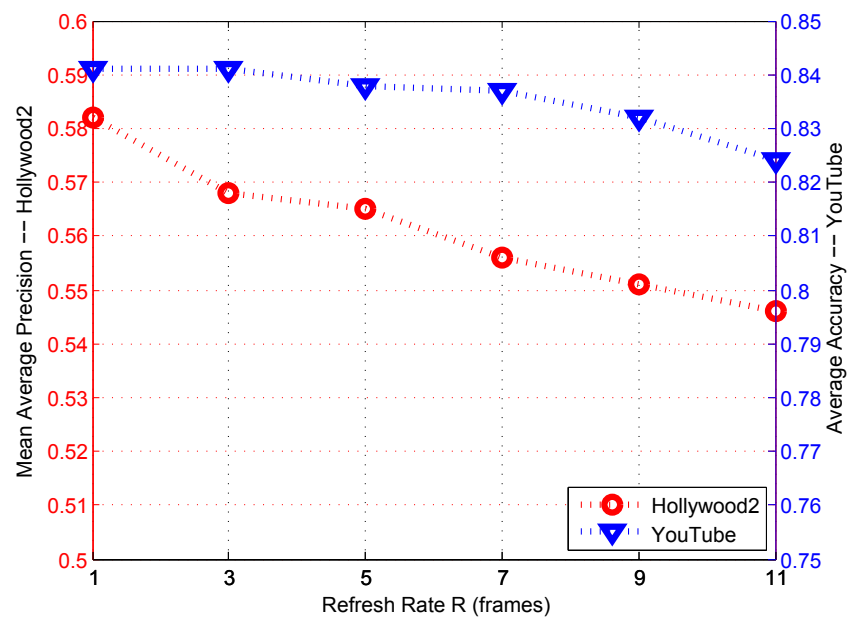

(f)

Fig. 11 Evaluation of the parameters on the Hollywood2 and YouTube datasets: (a) trajectory length, (b) sampling step size, (c) neighborhood size, (d) cell grid structure, (e) spatial scale number and (f) refresh rate. 
Results for various trajectory lengths are shown in Figure 11(a). For both datasets, increasing the length $L$ improves the performance up to $L=20$ frames. Trajectories need to have a minimum length in order to encode enough motion information. However, trajectories longer than 20 frames decrease the results, as they have a higher chance to drift from the initial position during the tracking process or to cross shot boundaries. We observe best results with a trajectory length of 15 or 20 frames.

With respect to the sampling step size $W$, Figure 11(b) presents the results for $W=2$ pixels to $W=20$ pixels. The performance increases with a higher sampling density. This is also consistent with dense sampling at regular positions where more features in general improve the results up to a point (Wang et al, 2009). For a step size of 2 (5) pixels, we report $58.9 \%$ (58.2\%) on Hollywood 2 and $84.4 \%$ (84.1\%) on YouTube. A sampling stride of 2 pixels samples every other pixel which significantly increases the computational complexity, see section 6.5. $W=5$ pixels offers a good trade-off between speed and accuracy.

Results are relatively stable with regard to the neighborhood size $N$, see Figure 11(c). On Hollywood2, the performance is comparable for values between $N=24$ and $N=48$ pixels. This is probably due to the descriptors which are strongly overlapping and thus form an over complete representation of the video. The best result on YouTube is $84.7 \%$ with a neighborhood size of 40 pixels.

Divisions of trajectory volumes into cells improve the results on both Hollywood 2 and YouTube. In particular, the performance increases significantly from a spatial cell grid $n_{\sigma}=1$ to $n_{\sigma}=2$, see Figure 11(d). Further increasing in the number of cells, i.e., beyond $n_{\sigma}=2, n_{\tau}=3$, does not yield better results. As best results, we report $58.5 \%$ on Hollywood 2 with a $2 \times 2 \times 2$ cell grid and $84.1 \%$ on YouTube with a $2 \times 2 \times 3$ cell grid.

Multiple spatial scales have been reported to improve the performance of local image descriptors. The same finding applies to our descriptors, see Figure 11(e). $S=1$ refers to only the original image scale. As $S$ increases, further scales are added. The improvement on Hollywood2 is over $2 \%$ from $56.1 \%$ to $58.2 \%$, whereas on YouTube it is $1.5 \%$ from $82.6 \%$ to $84.1 \%$. For both datasets the performance saturates at $S=5$ or $S=6$ spatial scales.

The refresh rate $R$ controls the frame rate, at which feature points are sampled in time. With the default setting, we sample new feature points in each frame. This may not be necessary for videos with high frame rate. We show results for different refresh rates $R$ in Figure 11(f). This parameter is more sensitive for Hollywood 2 than for YouTube. Movies often contain fast motion patterns, especially in action movies. It is then advantageous to sample feature points in each frame in order to capture more information. On the other hand, YouTube is very robust to the refresh rate $R$, the

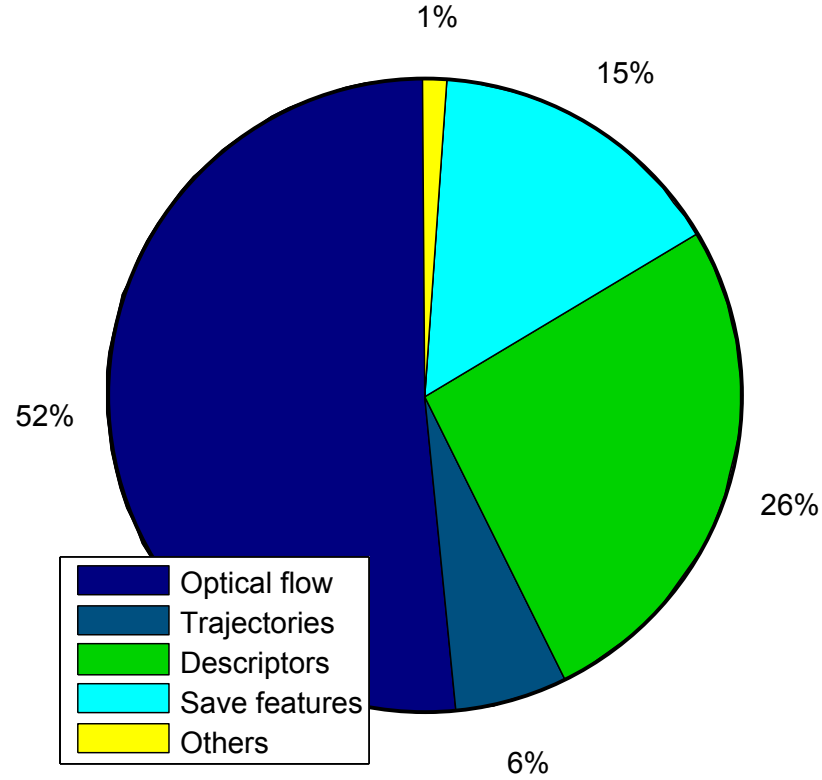

Fig. 12 The percentages of the time spent on the major steps of computing dense trajectories using the default parameter setting.

performance hardly changes up to a rate of $R=7$. This may be due to the temporal redundancy in YouTube videos and the missing shot boundaries.

\subsection{Computational complexity}

To analyze the computational complexity of the feature extraction, we compute dense trajectories for the 43 training video clips from the movie "American beauty" of the Hollywood 2 dataset. The resolution is $528 \times 224$ pixels, and the 43 video clips correspond to a total of approximately 15, 000 frames. We report run time (frames per second) and the number of features per frame in Figure 13. As a comparison, we also compute STIP (Harris3D+HOGHOF) features with the widely used STIP toolbox ${ }^{17}$ on the same videos. The run-time is obtained on a Dell server with a $2.6 \mathrm{GHz}$ quad-core Opteron CPU and 8GB RAM. We do not parallelize our code and only use a single core of the CPU.

Figure 12 analyzes the percentage of time spent on each step of computing dense trajectories for the default parameters. The computation of dense optical flow consumes most of the time with $52 \%$. Once the flow field is computed, tracking feature points is relatively inexpensive, it only takes $6 \%$ of the total computation time. Descriptor computation is the second most time-consuming step (26\%) with all four descriptors (Trajectory, HOG, HOF and MBH) being extracted. If only $\mathrm{MBH}$ descriptors are extracted, the descriptor computation time is reduce by $46 \%$. Note that the descriptor

17 http://www.irisa.fr/vista/Equipe/People/ Laptev/download.html 

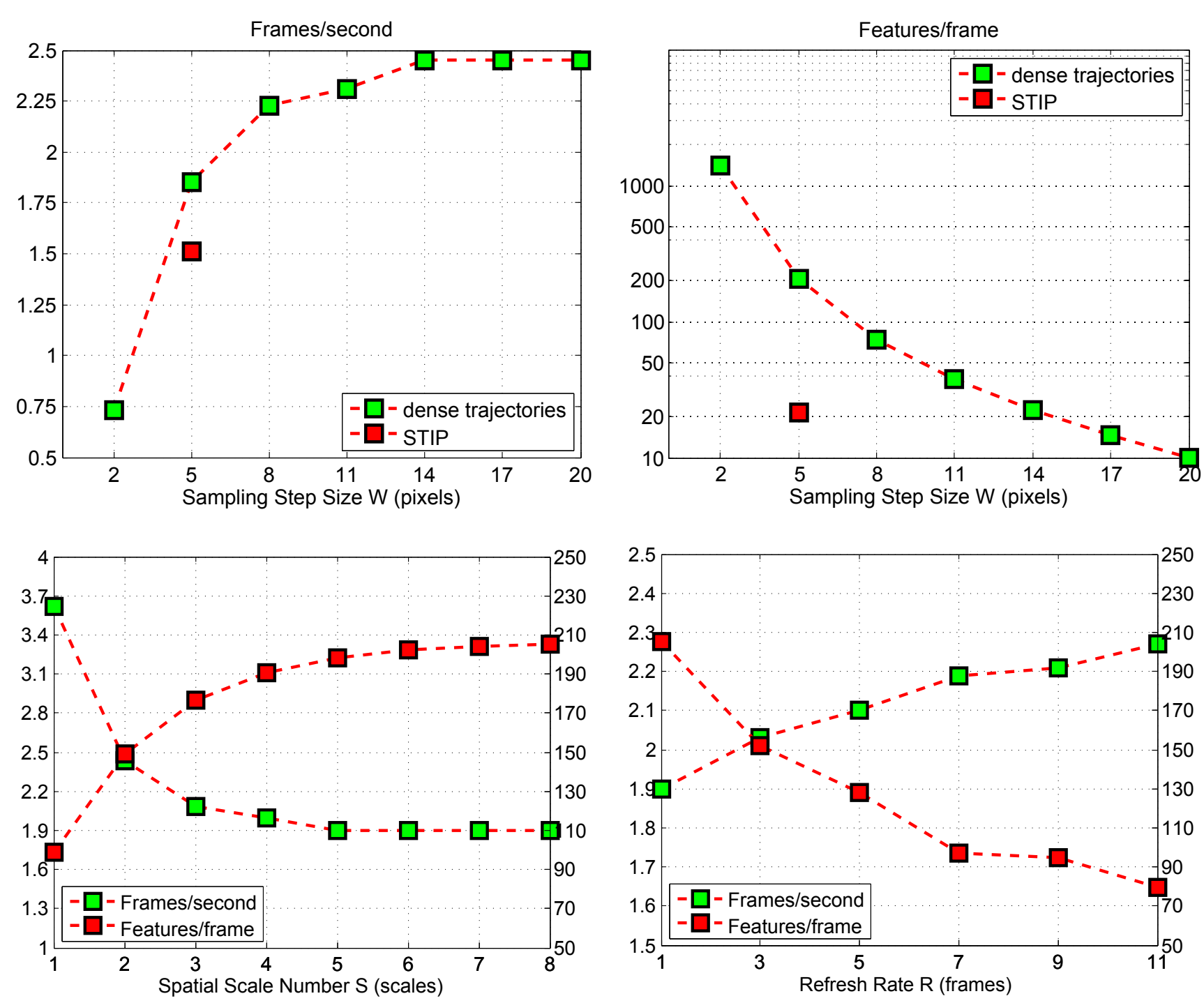

Fig. 13 The top row compares the computational complexity of dense trajectories and STIP (Laptev et al, 2008). We report run-time (top, left) and the number of features per frame (top, right). The bottom row presents an analysis of the complexity w.r.t. the spatial scale number $S$ (bottom, left) and refresh rate $R$ (bottom, right).

computation reuses the optical flow field which is only computed once. Finally, storing features to disk also takes time since our trajectories are dense.

The impact of the sampling step size $W$ on the computation time is depicted in the top row of Figure 13. The computational cost increases significantly for very low sampling step sizes. Here, the most expensive part is writing the features to the hard disk since we have 140 times more features for $W=2$ compared to $W=20$ pixels. We achieve 2.4 frames/second for $W=14$ pixels to $W=20$ pixels, in which case most of the time is used to compute dense optical flow fields. Using the default setting ( $W=5$ pixels), our code is slightly faster (1.8 frames/second) than the computation of STIP features (1.5 frames/second). However, we obtain about 10 times more features: 205.1 features/frame compared to 21.5 features/frame.

We illustrate the computational complexity for the spatial scale number $S$ and refresh rate $R$ in the bottom row of Figure 13. The speed is twice faster when features are only extracted on the original scale. The refresh rate $R$ has only a minor impact on the computational speed. We can reduce the number of features by either decreasing $S$ or increasing $R$.

\subsection{Comparison to state-of-the-art results}

In this section, we compare our results to the state of the art on each dataset. Table 7 displays our results with and 


\begin{tabular}{|c|c|c|c|c|c|}
\hline \multicolumn{2}{|l|}{ KTH } & \multicolumn{2}{|l|}{ YouTube } & \multicolumn{2}{|l|}{ Hollywood2 } \\
\hline Laptev et al (2008) & $91.8 \%$ & Liu et al (2009) & $71.2 \%$ & Wang et al (2009) & $47.7 \%$ \\
\hline Kovashka and Grauman (2010) & $94.53 \%$ & Ikizler-Cinbis and Sclaroff (2010) & $75.21 \%$ & Taylor et al (2010) & $46.6 \%$ \\
\hline Yuan et al (2011) & $93.7 \%$ & Brendel and Todorovic (2010) & $77.8 \%$ & Ullah et al (2010) & $53.2 \%$ \\
\hline Le et al (2011) & $93.9 \%$ & Le et al (2011) & $75.8 \%$ & Gilbert et al (2011) & $50.9 \%$ \\
\hline Gilbert et al (2011) & $94.5 \%$ & Bhattacharya et al (2011) & $76.5 \%$ & Le et al (2011) & $53.3 \%$ \\
\hline $\mathrm{MBH}$ & $95.0 \%$ & $\mathrm{MBH}$ & $80.6 \%$ & $\mathrm{MBH}$ & $55.1 \%$ \\
\hline Combined & $94.2 \%$ & Combined & $84.1 \%$ & Combined & $58.2 \%$ \\
\hline $\mathrm{MBH}+\mathrm{STP}$ & $95.3 \%$ & $\mathrm{MBH}+\mathrm{STP}$ & $83.0 \%$ & $\mathrm{MBH}+\mathrm{STP}$ & $57.6 \%$ \\
\hline Combined+STP & $94.4 \%$ & Combined+STP & $85.4 \%$ & Combined+STP & $59.9 \%$ \\
\hline \multicolumn{2}{|l|}{ UCF sports } & \multicolumn{2}{|l|}{ IXMAS } & \multicolumn{2}{|l|}{ Olympic Sports } \\
\hline Wang et al (2009) & $85.6 \%$ & Tran and Sorokin (2008) & $80.22 \%$ & Niebles et al (2010) & $72.1 \%$ \\
\hline Kläser et al (2010) & $86.7 \%$ & Junejo et al (2011) & $79.6 \%$ & Brendel and Todorovic (2011) & $77.3 \%$ \\
\hline Kovashka and Grauman (2010) & $87.27 \%$ & Wu et al (2011b) & $88.2 \%$ & Gaidon et al (2012) & $82.7 \%$ \\
\hline Le et al (2011) & $86.5 \%$ & & & & \\
\hline $\mathrm{MBH}$ & $84.2 \%$ & $\mathrm{MBH}$ & $91.8 \%$ & $\mathrm{MBH}$ & $71.6 \%$ \\
\hline Combined & $88.0 \%$ & Combined & $93.5 \%$ & Combined & $74.1 \%$ \\
\hline $\mathrm{MBH}+\mathrm{STP}$ & $84.0 \%$ & $\mathrm{MBH}+\mathrm{STP}$ & $91.9 \%$ & $\mathrm{MBH}+\mathrm{STP}$ & $74.9 \%$ \\
\hline Combined+STP & $89.1 \%$ & Combined+STP & $93.6 \%$ & Combined+STP & $77.2 \%$ \\
\hline \multicolumn{2}{|l|}{ UIUC } & \multicolumn{2}{|l|}{ UCF50 } & \multicolumn{2}{|l|}{ HMDB51 } \\
\hline Tran and Sorokin (2008) & $\mathbf{9 8 . 7 \%}$ & Kliper-Gross et al (2012) & $72.7 \%$ & Sadanand and Corso (2012) & $26.9 \%$ \\
\hline $\mathrm{MBH}$ & $97.1 \%$ & MBH & $82.2 \%$ & $\mathrm{MBH}$ & $43.2 \%$ \\
\hline Combined & $98.4 \%$ & Combined & $84.5 \%$ & Combined & $46.6 \%$ \\
\hline $\mathrm{MBH}+\mathrm{STP}$ & $98.1 \%$ & $\mathrm{MBH}+\mathrm{STP}$ & $83.6 \%$ & $\mathrm{MBH}+\mathrm{STP}$ & $45.1 \%$ \\
\hline Combined+STP & $98.3 \%$ & Combined+STP & $85.6 \%$ & Combined+STP & $48.3 \%$ \\
\hline
\end{tabular}

Table 7 Comparison of the MBH descriptor and a combination of all descriptors without and with spatio-temporal pyramids (STP) to the state of the art, as reported in the cited publications.

without spatio-temporal pyramids (STP) and compares our approach to the most recent results in the literature.

Spatio-temporal pyramids (STP) (Laptev et al, 2008) improve results on most datasets for both a single descriptor (e.g., MBH) and a combination of descriptors. With STP, the performance of $\mathrm{MBH}$ is improved by around $2 \%$ on YouTube, Hollywood 2 and HMDB51. For the combined result, the improvement is less significant. We observe about $1 \%$ of improvement on YouTube, Hollywood2, UCF sports, UCF50 and HMDB51. The biggest improvement is obtained for Olympic Sports, where both MBH and the combined result increase by about $3 \%$ when using the spatio-temporal pyramid. There is no improvement for datasets with clean background (i.e., KTH, IXMAS, UIUC).
Table 7 also compares our approach to state-of-the-art results. We can observe that it outperforms significantly the state of the art on all datasets except KTH, UIUC, and Olympic Sports where it is on par with results in the literature.

On YouTube, we significantly outperform the state of the art (Brendel and Todorovic, 2010) by over 8\%. On Hollywood2 "combined+STP" reports $59.9 \%$. This is over $6 \%$ higher than the state of the art (Le et al, 2011) that employs features learning using deep networks. On UCF Sports "combined+STP" achieves $89.1 \%$ which is around $2 \%$ better than the state of the art (Kovashka and Grauman, 2010).

IXMAS is a popular multi-view action dataset. There are various experimental settings designed for cross-view action recognition. We compare to the results which are also 
trained and tested on all five views. "Combined+STP" reports $93.6 \%$ and significantly outperforms the state of the art (Wu et al, 2011b) by $5 \%$. Weinland et al (2006) reported $93.33 \%$ using motion history volumes of 3D human body models for training and testing. These 3D exemplars were obtained based on reconstruction given the five views.

Niebles et al (2010) obtain $72.1 \%$ on Olympic Sports with an approach which models the temporal structure of a human action. Note that we get a similar result (i.e., 71.6\%) with a single $\mathrm{MBH}$ descriptor. "Combined+STP" reports $77.2 \%$ which outperforms their result by over $5 \%$. Brendel and Todorovic (2011) reported a similar result $(77.3 \%)$ by designing complex spatio-temporal graphs to model the hierarchical relationships embedded in actions. Recently, Gaidon et al (2012) achieved $82.7 \%$ by clustering dense trajectories and modeling the relationship between the clusters via a tree structure.

On UCF50, "MBH + STP" results in $83.6 \%$, whereas "Combined+STP" achieves 85.6\%. Recently, Kliper-Gross et al (2012) reported $72.7 \%$ by designing descriptors to capture local changes in motion directions. On the recent HMDB5 dataset, we outperform the state of the art (Sadanand and Corso, 2012) by $16 \%$ using only "MBH". "Combined+STP" further improves the performance by $5 \%$.

\section{Conclusions}

This paper introduced an approach for efficient video description based on dense trajectories and motion boundary histogram descriptors. Our dense trajectories have shown to outperform previous approaches that extract trajectories with either the Kanade-Lucas-Tomasi (KLT) tracker or by SIFT descriptor matching. Motion boundary histogram descriptors which are computed along the dense trajectories have shown to yield excellent results. They are designed to be robust to camera motion and are shown to outperform the state-of-the-art histogram of optical flow descriptor. We have evaluated our video description extensively on nine datasets and have shown that it significantly outperforms the state of the art.

Our video description is by all means not limited to a bag-of-features representation and could be applied in the context of action localization and video retrieval. The efficiency of the algorithm enables its application on large scale video data. Yet, its performance is currently limited by the quality of the optical flow available. The state-of-the-art optical flow algorithms are often computationally expensive and far from perfect, as discussed in section 6.3. Developing better optical flow algorithms suitable for large realistic video datasets is important to improve the performance of current action recognition systems.
Acknowledgements This work was supported in part by the National Natural Science Foundation of China (NSFC) Grant 60825301, the National Basic Research Program of China (973 Program) Grant 2012CB316302, the Strategic Priority Research Program of the Chinese Academy of Sciences (Grant XDA06030300), as well as the joint Microsoft/INRIA project and the European integrated project AXES.

\section{References}

Anjum N, Cavallaro A (2008) Multifeature object trajectory clustering for video analysis. IEEE Transactions on Multimedia 18:1555-1564

Bay H, Tuytelaars T, Gool LV (2006) SURF: Speeded up robust features. In: European Conference on Computer Vision

Bhattacharya S, Sukthankar R, Jin R, Shah M (2011) A probabilistic representation for efficient large scale visual recognition tasks. In: IEEE Conference on Computer Vision and Pattern Recognition

Bregonzio M, Gong S, Xiang T (2009) Recognising action as clouds of space-time interest points. In: IEEE Conference on Computer Vision and Pattern Recognition

Brendel W, Todorovic S (2010) Activities as time series of human postures. In: European Conference on Computer Vision

Brendel W, Todorovic S (2011) Learning spatiotemporal graphs of human activities. In: IEEE International Conference on Computer Vision

Brox T, Malik J (2010) Object segmentation by long term analysis of point trajectories. In: European Conference on Computer Vision

Brox T, Malik J (2011) Large displacement optical flow: Descriptor matching in variational motion estimation. IEEE Transactions on Pattern Analysis and Machine Intelligence 33(3):500-513

Dalal N, Triggs B (2005) Histograms of oriented gradients for human detection. In: IEEE Conference on Computer Vision and Pattern Recognition

Dalal N, Triggs B, Schmid C (2006) Human detection using oriented histograms of flow and appearance. In: European Conference on Computer Vision

Dollár P, Rabaud V, Cottrell G, Belongie S (2005) Behavior recognition via sparse spatio-temporal features. In: IEEE Workshop Visual Surveillance and Performance Evaluation of Tracking and Surveillance

Farnebäck G (2003) Two-frame motion estimation based on polynomial expansion. In: Proceedings of the Scandinavian Conference on Image Analysis

Fei-Fei L, Perona P (2005) A Bayesian hierarchical model for learning natural scene categories. In: IEEE Conference on Computer Vision and Pattern Recognition

Gaidon A, Harchaoui Z, Schmid C (2012) Recognizing activities with cluster-trees of tracklets. In: British Machine Vision Conference

Gilbert A, Illingworth J, Bowden R (2011) Action recognition using mined hierarchical compound features. IEEE Transactions on Pattern Analysis and Machine Intelligence 33(5):883-897

Hervieu A, Bouthemy P, Cadre JPL (2008) A statistical video content recognition method using invariant features on object trajectories. IEEE Transactions on Circuits and Systems for Video Technology 18:1533-1543

Ikizler-Cinbis N, Sclaroff S (2010) Object, scene and actions: combining multiple features for human action recognition. In: European Conference on Computer Vision

Johnson N, Hogg D (1996) Learning the distribution of object trajectories for event recognition. Image and Vision Computing 14:609-615

Junejo IN, Dexter E, Laptev I, Pérez P (2011) View-independent action recognition from temporal self-similarities. IEEE Transactions on Pattern Analysis and Machine Intelligence 33(1):172-185

Jung CR, Hennemann L, Musse SR (2008) Event detection using trajectory clustering and 4-D histograms. IEEE Transactions on Circuits and Systems for Video Technology 18:1565-1575 
Kläser A, Marszałek M, Schmid C (2008) A spatio-temporal descriptor based on 3D-gradients. In: British Machine Vision Conference

Kläser A, Marszałek M, Laptev I, Schmid C (2010) Will person detection help bag-of-features action recognition? Tech. Rep. RR-7373, INRIA

Kliper-Gross O, Gurovich Y, Hassner T, Wolf L (2012) Motion interchange patterns for action recognition in unconstrained videos. In: European Conference on Computer Vision

Kovashka A, Grauman K (2010) Learning a hierarchy of discriminative space-time neighborhood features for human action recognition. In: IEEE Conference on Computer Vision and Pattern Recognition

Kuehne H, Jhuang H, Garrote E, Poggio T, Serre T (2011) Hmdb: A large video database for human motion recognition. In: IEEE International Conference on Computer Vision, IEEE, pp 2556-2563

Laptev I (2005) On space-time interest points. International Journal of Computer Vision 64(2-3):107-123

Laptev I, Marszałek M, Schmid C, Rozenfeld B (2008) Learning realistic human actions from movies. In: IEEE Conference on Computer Vision and Pattern Recognition

Lazebnik S, Schmid C, Ponce J (2006) Beyond bags of features: Spatial pyramid matching for recognizing natural scene categories. In: IEEE Conference on Computer Vision and Pattern Recognition

Le QV, Zou WY, Yeung SY, Ng AY (2011) Learning hierarchical invariant spatio-temporal features for action recognition with independent subspace analysis. In: IEEE Conference on Computer Vision and Pattern Recognition

Liu J, Luo J, Shah M (2009) Recognizing realistic actions from videos in the wild. In: IEEE Conference on Computer Vision and Pattern Recognition

Lowe DG (2004) Distinctive image features from scale-invariant keypoints. International Journal of Computer Vision 60(2):91-110

Lu WC, Wang YCF, Chen CS (2010) Learning dense optical-flow trajectory patterns for video object extraction. In: IEEE Advanced Video and Signal Based Surveillance Conference

Lucas BD, Kanade T (1981) An iterative image registration technique with an application to stereo vision. In: International Joint Conference on Artificial Intelligence

Marszałek M, Laptev I, Schmid C (2009) Actions in context. In: IEEE Conference on Computer Vision and Pattern Recognition

Matikainen P, Hebert M, Sukthankar R (2009) Trajectons: Action recognition through the motion analysis of tracked features. In: ICCV Workshops on Video-Oriented Object and Event Classification

Messing R, Pal C, Kautz H (2009) Activity recognition using the velocity histories of tracked keypoints. In: IEEE International Conference on Computer Vision

Niebles JC, Chen CW, Fei-Fei L (2010) Modeling temporal structure of decomposable motion segments for activity classification. In: European Conference on Computer Vision

Nowak E, Jurie F, Triggs B (2006) Sampling strategies for bag-offeatures image classification. In: European Conference on Computer Vision

Ojala T, Pietikainen M, Maenpaa T (2002) Multiresolution gray-scale and rotation invariant texture classification with local binary patterns. IEEE Transactions on Pattern Analysis and Machine Intelligence 24(7):971-987

Piriou G, Bouthemy P, Yao JF (2006) Recognition of dynamic video contents with global probabilistic models of visual motion. IEEE Transactions on Image Processing 15:3418-3431

Raptis M, Soatto S (2010) Tracklet descriptors for action modeling and video analysis. In: European Conference on Computer Vision

Reddy K, Shah M (2012) Recognizing 50 human action categories of web videos. Machine Vision and Applications pp 1-11

Rodriguez MD, Ahmed J, Shah M (2008) Action MACH a spatiotemporal maximum average correlation height filter for action recognition. In: IEEE Conference on Computer Vision and Pattern
Recognition

Sadanand S, Corso JJ (2012) Action bank: A high-level representation of activity in video. In: IEEE Conference on Computer Vision and Pattern Recognition

Sand P, Teller S (2008) Particle video: Long-range motion estimation using point trajectories. International Journal of Computer Vision 80:72-91

Schüldt C, Laptev I, Caputo B (2004) Recognizing human actions: a local SVM approach. In: International Conference on Pattern Recognition

Scovanner P, Ali S, Shah M (2007) A 3-dimensional SIFT descriptor and its application to action recognition. In: ACM Conference on Multimedia

Shi J, Tomasi C (1994) Good features to track. In: IEEE Conference on Computer Vision and Pattern Recognition

Sun J, Wu X, Yan S, Cheong LF, Chua TS, Li J (2009) Hierarchical spatio-temporal context modeling for action recognition. In: IEEE Conference on Computer Vision and Pattern Recognition

Sun J, Mu Y, Yan S, Cheong LF (2010) Activity recognition using dense long-duration trajectories. In: IEEE International Conference on Multimedia and Expo

Sundaram N, Brox T, Keutzer K (2010) Dense point trajectories by GPU-accelerated large displacement optical flow. In: European Conference on Computer Vision

Taylor GW, Fergus R, LeCun Y, Bregler C (2010) Convolutional learning of spatio-temporal features. In: European Conference on Computer Vision

Tran D, Sorokin A (2008) Human activity recognition with metric learning. In: European Conference on Computer Vision

Uemura H, Ishikawa S, Mikolajczyk K (2008) Feature tracking and motion compensation for action recognition. In: British Machine Vision Conference

Ullah MM, Parizi SN, Laptev I (2010) Improving bag-of-features action recognition with non-local cues. In: British Machine Vision Conference

Wang H, Ullah MM, Kläser A, Laptev I, Schmid C (2009) Evaluation of local spatio-temporal features for action recognition. In: British Machine Vision Conference

Wang H, Kläser A, Schmid C, Liu CL (2011) Action recognition by dense trajectories. In: IEEE Conference on Computer Vision and Pattern Recognition

Wang X, Ma KT, Ng GW, Grimson WEL (2008) Trajectory analysis and semantic region modeling using a nonparametric Bayesian model. In: IEEE International Conference on Computer Vision

Weinland D, Ronfard R, Boyer E (2006) Free viewpoint action recognition using motion history volumes. Computer Vision and Image Understanding 104(2):249-257

Weinland D, Boyer E, Ronfard R (2007) Action recognition from arbitrary views using $3 \mathrm{D}$ exemplars. In: IEEE International Conference on Computer Vision

Willems G, Tuytelaars T, Gool L (2008) An efficient dense and scaleinvariant spatio-temporal interest point detector. In: European Conference on Computer Vision

Wong SF, Cipolla R (2007) Extracting spatiotemporal interest points using global information. In: IEEE International Conference on Computer Vision

Wu S, Oreifej O, Shah M (2011a) Action recognition in videos acquired by a moving camera using motion decomposition of lagrangian particle trajectories. In: IEEE International Conference on Computer Vision

Wu X, Xu D, Duan L, Luo J (2011b) Action recognition using context and appearance distribution features. In: IEEE Conference on Computer Vision and Pattern Recognition

Yeffet L, Wolf L (2009) Local trinary patterns for human action recognition. In: IEEE International Conference on Computer Vision 
Yuan J, Liu Z, Wu Y (2011) Discriminative video pattern search for efficient action detection. IEEE Transactions on Pattern Analysis and Machine Intelligence 33:1728-1743

Zhang J, Marszałek M, Lazebnik S, Schmid C (2007) Local features and kernels for classification of texture and object categories: A comprehensive study. International Journal of Computer Vision 73(2):213-238 